\title{
Gene expression variation in Arabidopsis embryos at single-nucleus resolution
}

\author{
Ping Kao ${ }^{1}$, Michael A. Schon ${ }^{1}$, Magdalena Mosiolek ${ }^{1}$, Balaji Enugutti ${ }^{1}$ and Michael D. Nodine ${ }^{1,2, *}$
}

\begin{abstract}
Soon after fertilization of egg and sperm, plant genomes become transcriptionally activated and drive a series of coordinated cell divisions to form the basic body plan during embryogenesis. Early embryonic cells rapidly diversify from each other, and investigation of the corresponding gene expression dynamics can help elucidate underlying cellular differentiation programs. However, current plant embryonic transcriptome datasets either lack cell-specific information or have RNA contamination from surrounding non-embryonic tissues. We have coupled fluorescence-activated nuclei sorting together with single-nucleus mRNA-sequencing to construct a gene expression atlas of Arabidopsis thaliana early embryos at single-cell resolution. In addition to characterizing cell-specific transcriptomes, we found evidence that distinct epigenetic and transcriptional regulatory mechanisms operate across emerging embryonic cell types. These datasets and analyses, as well as the approach we devised, are expected to facilitate the discovery of molecular mechanisms underlying pattern formation in plant embryos.
\end{abstract}

This article has an associated 'The people behind the papers' interview.

KEY WORDS: Arabidopsis, Embryo, Single-nucleus RNA-seq, Gene expression, Transcription factor, Epigenetic

\section{INTRODUCTION}

Metazoans and land plants establish their body plans during embryogenesis (Dresselhaus and Jürgens, 2021; Gerri et al., 2020), and corresponding gene regulatory mechanisms have evolved independently in these two major eukaryotic lineages to help generate the immense morphological diversity observed in nature (Bai, 2015; Clark et al., 2006; Meyerowitz, 2002). For example, in animals it has been long recognized that maternal gene products control initial pattern formation before the transition of control from the maternal to the zygotic genome (Lee et al., 2014; Tadros and Lipshitz, 2009). By contrast, transcriptional activation of the zygotic genome soon after fertilization is necessary for zygote elongation and initial divisions in Nicotiana tabacum (tobacco) (Zhao et al.,

\footnotetext{
${ }^{1}$ Gregor Mendel Institute (GMI), Austrian Academy of Sciences, Vienna Bio Center (VBC), Dr Bohr-Gasse 3, 1030 Vienna, Austria. ${ }^{2}$ Laboratory of Molecular Biology, Wageningen University, Wageningen 6708 PB, The Netherlands.

*Author for correspondence (michael.nodine@wur.nl)

(iD M.A.S., 0000-0002-4756-3906; M.M., 0000-0002-2204-1298; B.E., 0000-00020816-024X; M.D.N., 0000-0002-6204-8857

This is an Open Access article distributed under the terms of the Creative Commons Attribution License (https://creativecommons.org/licenses/by/4.0), which permits unrestricted use,

distribution and reproduction in any medium provided that the original work is properly attributed.

Handling Editor: Ykä Helariutta

Received 5 March 2021; Accepted 24 May 2021
}

2011) and the model flowering plant Arabidopsis thaliana (Arabidopsis) (Kao and Nodine, 2019; Zhao et al., 2019). In addition, the vast majority of genes regulating Arabidopsis embryo morphogenesis are zygotically expressed (Nodine and Bartel, 2012; Zhao et al., 2019) and required (Meinke, 2020; Muralla et al., 2011). Therefore, genes are expressed from the zygotic genome during initial stages of embryo development, and the diversification of gene expression programs across plant embryonic cell types contributes to the formation of the basic body plan. Characterizing how gene expression programs are established in individual cell types of early embryos is crucial to understand the molecular basis of pattern formation in plant embryos, and more broadly the general and unique principles of embryonic patterning in multicellular organisms.

Forward genetic screens successfully identified many genes that are required for proper plant embryogenesis (Lukowitz et al., 2004; Mayer et al., 1998; Meinke, 2020), but relatively few mutations in genes encoding cell-specific transcriptional regulators were recovered. This is at least partially due to the high degree of genetic redundancy among plant transcription factors (TFs) that typically belong to multigene families (Riechmann, 2002). As an alternative approach, RNA populations can be characterized to infer gene-regulatory processes underlying cellular differentiation events. Transcriptomes generated from early embryos at various stages of development have accordingly yielded insights into the biological processes operating during different embryonic phases (Belmonte et al., 2013; Hofmann et al., 2019; Xiang et al., 2011; Zhao et al., 2019). However, these transcriptomes were generated from whole embryos. Additional studies have revealed genes that are preferentially expressed in broad (Belmonte et al., 2013; Casson et al., 2005; Chen et al., 2021; Slane et al., 2014; Zhou et al., 2020) or more specific (Palovaara et al., 2017) regions of plant embryos, but either lack cellular resolution or were contaminated with RNAs derived from the maternal seed coat that encompasses the developing embryo (Schon and Nodine, 2017).

Single-cell mRNA-sequencing (scRNA-seq) has been instrumental towards understanding developmental events at cellular resolution over the past decade (Chen et al., 2019; Hwang et al., 2018). Several studies have applied these approaches to plant tissues (Brennecke et al., 2013; Efroni and Birnbaum, 2016; JeanBaptiste et al., 2019; Ryu et al., 2019; Satterlee et al., 2020; Shulse et al., 2019; Song et al., 2020; Xu et al., 2021; Zhang et al., 2019), but scRNA-seq has yet to be reported for individual cell types in plant embryos. This is primarily due to the presence of rigid cell walls that hold plant cells together. Although cell walls can be removed by enzymatic treatment of tissues that are easy to access, such protoplasting techniques remain impractical for early embryos because they are deeply embedded within maternal seed tissues. Single-nucleus mRNA-sequencing (snRNA-seq) (Habib et al., 2016) offers an alternative method to inspect transcriptomes at single-cell resolution in plants and has been recently applied to 
A
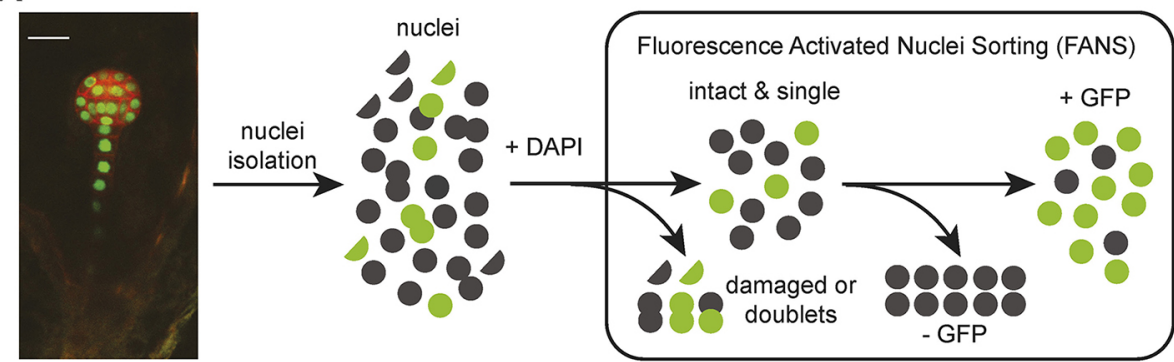

B

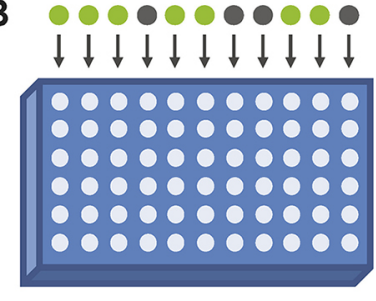

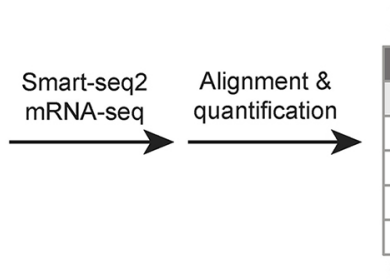

$\begin{array}{llllll}0 & 10 & 20 & 30 & 40 & 50\end{array}$

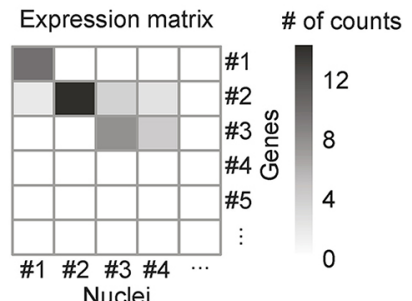

Nuclei

C

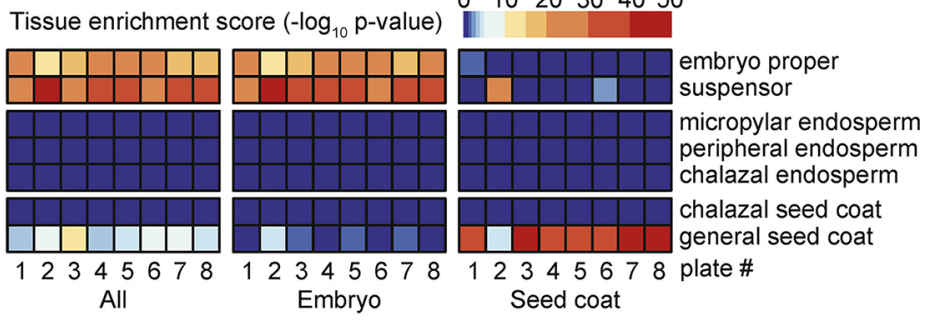

Fig. 1. Acquisition of contamination-free transcriptomes from individual embryonic nuclei. (A) Schematic showing collection of single embryonic nuclei. GFP-positive fixed nuclei from pWOX2::NLS-GFP transgenic developing seeds were sorted and collected by FANS. Scale bar: $20 \mu \mathrm{m}$. (B) Diagram showing how single-nucleus libraries were generated with a modified Smartseq2 protocol (Picelli et al., 2014a,b), sequenced and individual gene expression quantified. (C) Maternal contamination assessment and removal. Nuclei from each plate were assigned as embryonic or seed-coat-derived according to the unsupervised clustering and tissue enrichment tests (Fig. S2A-C). Tissue enrichment tests based on the mean expression of all nuclei or nuclei categorized as embryo or seed coat are shown. roots (Farmer et al., 2021) and endosperm tissues within seeds (Long et al., 2021; Picard et al., 2021). Here, we present a workflow to obtain contamination-free high-quality transcriptomes from individual early embryonic nuclei followed by their assignments to the precursors of the most fundamental plant tissues including the shoot meristem, distal regions of the root meristem and epidermal and vascular tissues. Remarkably, these initial embryonic cell types already express characteristic sets of genes, have different evolutionary trajectories and appear to be regulated by distinct epigenetic and transcriptional mechanisms.

\section{RESULTS}

\section{Acquisition of contamination-free transcriptomes from} individual embryonic nuclei

To acquire single-cell transcriptomes of early Arabidopsis embryos, we used fluorescence-activated nuclei sorting (FANS) coupled with snRNA-seq (Fig. 1A,B). More specifically, we used a transgenic line expressing nuclear-localized green fluorescent protein (GFP) under the control of the embryo-specific WUSCHEL-RELATED HOMEOBOX 2 (WOX2) promoter (pWOX2::H2B-GFP, pWOX2:: tdTomato-LTI6b; hereafter referred to as pWOX2::NLS-GFP) to fluorescently label nuclei in embryos but not the surrounding endosperm or maternal tissues (Fig. 1A) (Gooh et al., 2015). We chose to focus on globular-stage embryos because this is when the precursors to the most fundamental plant tissues emerge along apicalbasal and radial embryonic axes (Palovaara et al., 2016). Briefly, we fixed siliques or seeds containing globular-stage embryos with a low concentration of dithiobis (succinimidyl propionate) (DSP) before nuclei isolation to preserve RNA. Nuclei were also stained with 4',6diamidino-2-phenylindole (DAPI), and intact nuclei were selected based on DAPI profiles (Fig. S1A,C). Embryonic nuclei were then isolated based on their strong GFP signal (Fig. S1B,D) and sorted individually into 96-well plates. Fixed nuclei were decrosslinked with dithiothreitol (DTT) to enable the generation of cDNA and the Smartseq2 protocol (Picelli et al., 2014a,b) was used to construct nextgeneration sequencing (NGS)-compatible libraries. NGS libraries were then sequenced on an Illumina HiSeq 2500 followed by the alignment of NGS reads to the Araport11 transcriptome (Cheng et al., 2017) and transcript quantification by Kallisto (Bray et al., 2016) (Fig. 1B). After quality controls (see Materials and Methods), 534 out of $744(72 \%)$ nuclei were retained for further analyses. A total of 24,591 genes were detected from all nuclei with an average of 440,289 aligned reads and 2576 detected genes per snRNA-seq library (Fig. S1E,F; Table S1). Therefore, our approach allowed us to acquire high-quality RNA-seq libraries from hundreds of individual embryonic nuclei.

Contamination of early embryonic mRNA-seq datasets with RNAs from surrounding maternal seed tissues has been a major limitation to embryo transcriptomics (Schon and Nodine, 2017). To evaluate the level of maternal contamination in individual snRNA-seq libraries, we applied the tissue enrichment test (Schon and Nodine, 2017). Although we attempted to achieve $99.9 \%$ accuracy with our stringent FANS selection (Fig. S1), embryonic nuclei comprised only $0.1-1 \%$ of seed nuclei and thus false positive events were non-negligible and further filtering was required. Accordingly, $20-50 \%$ of the snRNA-seq libraries per plate were significantly enriched for either seed coat or endosperm transcripts, whereas remaining snRNA-seq libraries were enriched for embryonic transcripts or had ambiguous identities (Fig. S2A). To systematically identify contaminated snRNA-seq libraries, we conducted unsupervised clustering on all libraries and labeled them according to their tissue enrichment scores (Fig. S2B,C). Because clusters 12 and 13 were enriched for libraries with seed coat contamination, we excluded them from subsequent analyses 
A

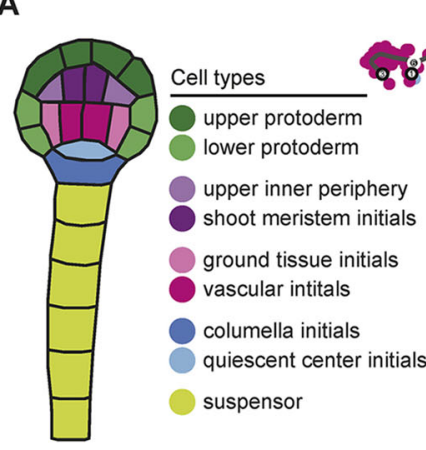

C

Expressing cell types

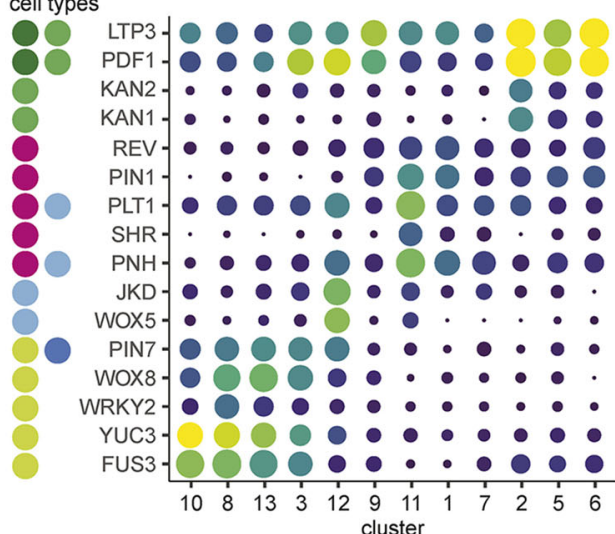

$\mathbf{E}$

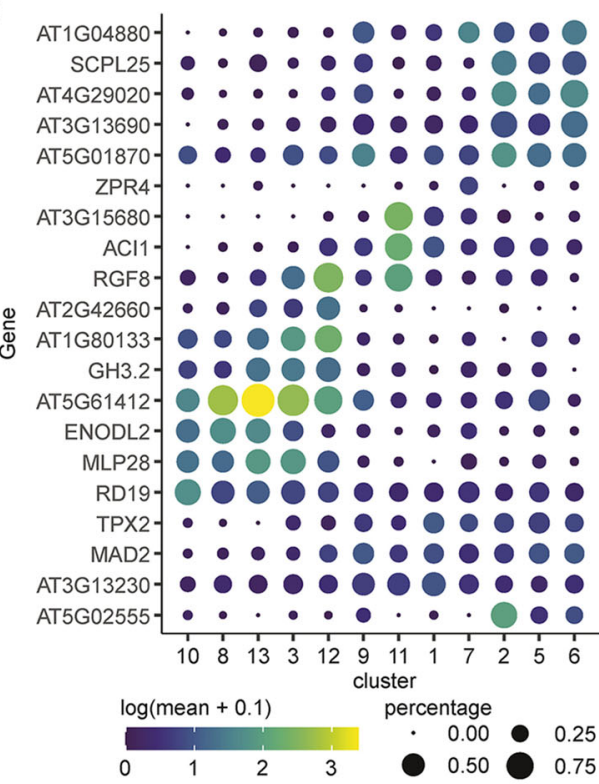

B

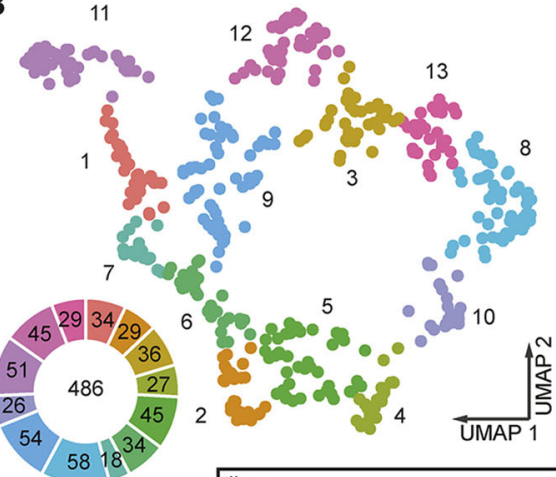

\# D

D

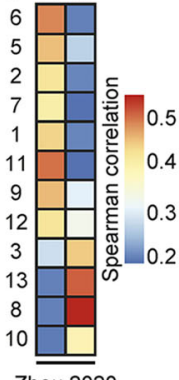

Zhou 2020

$\mathbf{F}$

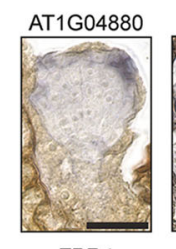

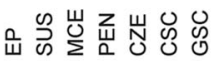

\#

Cell-type assignment

6 upper protoderm (upd)

5 dividing outer (div.0)

2 lower protoderm (Ipd)

7 shoot meristem initials (smi)

1 dividing inner (div.i)

11 vascular initials (vas)

9 dividing cells (div)

$12 \bigcirc$ quiescent center initials (qc)

3 columella initials (col)

13 suspensor (sus3)

8 suspensor (sus2)

$10 \bigcirc$ suspensor (sus1)

Belmonte 2013
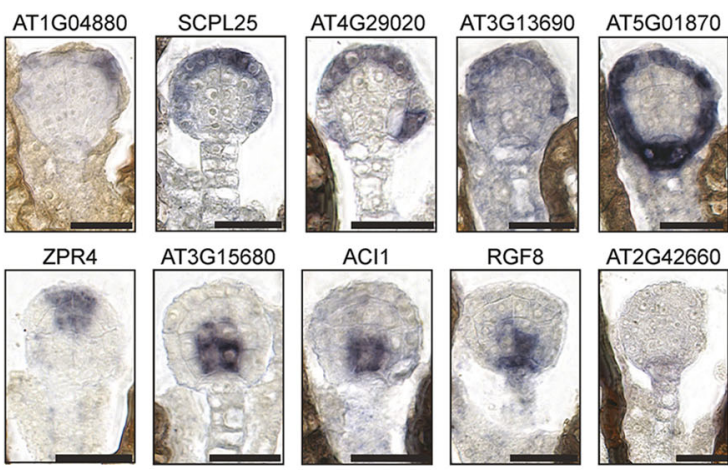

AT3G15680

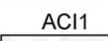

RGF8

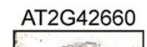

AT1G80133

$\mathrm{GH} 3.2$
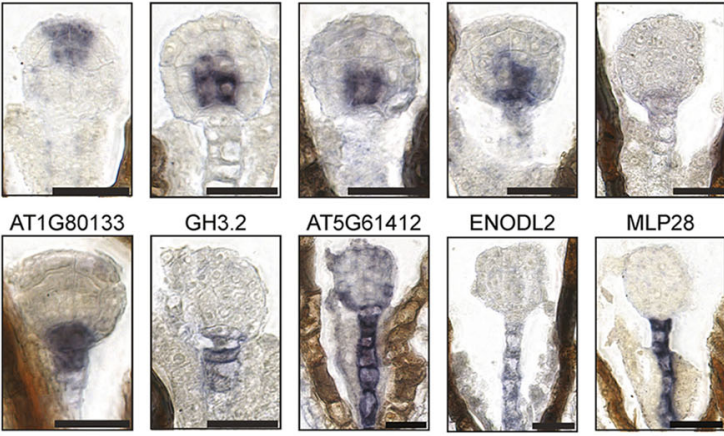

ENODL2

RD19

TPX2

MAD2

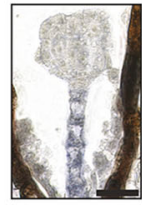

MLP28
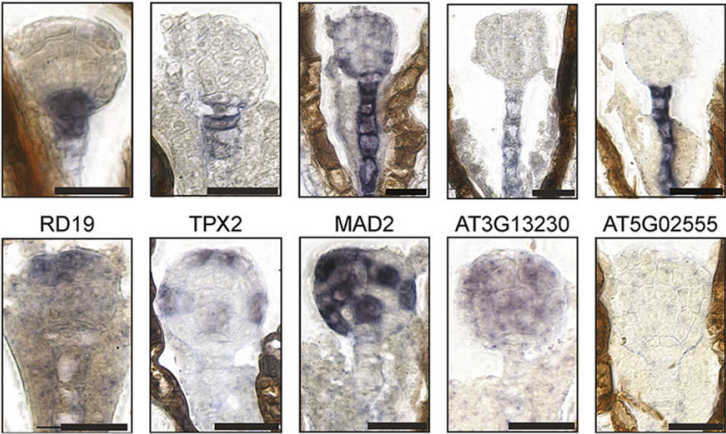

AT5G02555

Fig. 2. Identification of embryonic cell types. (A) Resolving nine defined cell types by supervised clustering. Marker genes expressed in at least one of the nine cell types were used to calculate cell-type scores with hypergeometric tests. Each dot represents a nucleus and nuclei were labeled according to the cell type with the highest cell-type score. (B) The thirteen clusters corresponding to A. The number of nuclei for each cluster is indicated in the donut plot. (C) Dot plot illustrating expression patterns of known cell type-specific markers. The cell types in which a marker gene is expressed were color-coded according to A. The sizes of dots represent the percentage of nuclei in which the transcript was detected for each cluster and the colors represent the log ${ }_{10}$-transformed mean expression levels of each cluster. (D) Spearman's correlation coefficients between cluster mean expression and published globular-stage embryo proper (32E) and suspensor (32S) transcriptomes (Zhou et al., 2020) (left). Tissue enrichment test results based on cluster mean expression using published transcriptomes from seed tissues as a reference (Belmonte et al., 2013; Schon and Nodine, 2017) (right). EP, embryo proper; SUS, suspensor; MCE, micropylar endosperm; PEN, peripheral

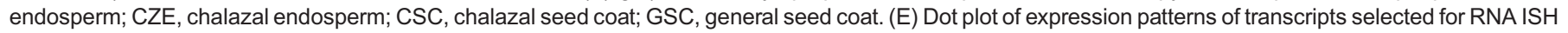

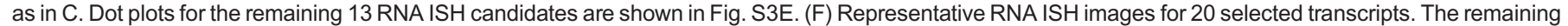
13 RNA ISH candidates are presented in Fig. S3F. Scale bars: $20 \mu \mathrm{m}$. Quantification of RNA ISH images is shown in Fig. S4. (G) Assigned cell types and abbreviations for clusters. 
(Fig. S2B,C). To further evaluate how well we could remove non-embryonic nuclei, we combined the expression levels of snRNA-seq libraries from each plate and performed tissue enrichment tests (Fig. 1C). Retained and excluded nuclei were enriched for embryonic and seed coat cell types, respectively. Moreover, transcriptomes from the retained nuclei were more similar to published embryonic transcriptomes (Hofmann et al., 2019; Nodine and Bartel, 2012) than those from discarded nuclei (Fig. S2C). Altogether, our stringent criteria allowed us to successfully remove non-embryonic snRNA-seq libraries and obtain 486 high-quality snRNA-seq libraries from embryonic cells.

\section{Identification of embryonic cell types}

Unsupervised uniform manifold approximation and projection (UMAP) clustering of snRNA-seq libraries was able to distinguish embryo proper and suspensor nuclei, but not individual cell types (Fig. S3A). The inability of unsupervised UMAP clustering to resolve individual cell types could be due to the relatively low number of snRNA-seq libraries used $(n=486)$ or the transient fates of early embryonic cell types. Nevertheless, as an alternative to unsupervised clustering we used enrichments and depletions of known cell-specific transcripts in each nucleus to determine how likely it was for each nucleus to come from each cell type. We first identified 174 reference genes expressed in embryos from the literature and recorded their expression patterns as either expressed or not expressed in the cell types present in globular embryos (Table S2; Fig. 2A). More specifically, nine cell types are found in globular embryos (Palovaara et al., 2016) and can be classified based on whether they derive from the larger basal cell or smaller apical cell formed upon zygote division. The corresponding basal cell lineage (BCL) consists of the terminally differentiated suspensor, which connects maternal tissues with the embryo proper, as well as the columella and quiescent center initials, which are precursors to distal regions of the root meristem. Unlike the BCL, the apical cell lineage (ACL) divides along the radial embryonic axis to form concentric tissue layers. The outermost protoderm, middle ground tissue initials and innermost vascular initials produce the epidermal, ground and vascular tissues, respectively; whereas the shoot meristem initials will produce aerial tissues after germination. Presence or absence of these reference genes were then used in hypergeometric tests to compute cell-type scores for each nucleus of these nine cell types in all 486 snRNA-seq libraries (see Materials and Methods). We then performed UMAP clustering on the cell-type scores, and identified 12 clusters that were each enriched for a specific cell type (Fig. 2A,B). We also identified one cluster (cluster 4) that was enriched for multiple cell types and had substantially fewer genes detected per snRNA-seq library compared with the other clusters. We discarded the snRNA-seq libraries belonging to this cluster from subsequent analyses because of their poor quality, which may be due to being generated from aggregated or fragmented nuclei. Cell-specific reference transcripts tended to co-localize to the same cluster (Fig. 2C; Fig. S3B) indicating that clustering on cell-type scores recapitulates expression patterns of reference markers. For example, WOX5 and JACKDAW (JKD) transcripts are highly enriched in the quiescent center initials (Haecker et al., 2004; Welch et al., 2007) and co-localize to cluster 12 . Therefore, by highlighting the differences among cell types based on a reference gene set we were able to resolve the 486 snRNA-seq libraries into 12 clusters representing distinct cell types.

To independently test these marker-based predictions, we compared the transcriptomes of each cell cluster with published transcriptomes from the embryo proper and suspensor regions of globular embryos (Belmonte et al., 2013). In agreement with the marker-based assignments, clusters 8, 10 and 13 were exclusively enriched for suspensor transcripts based on tissue enrichment tests (Fig. 2D). Also consistent with the cell type assignments, clusters 1, 2, 5, 6, 7 and 11 were enriched for only embryo proper transcripts. Cluster 9 had mixed cell type assignments and accordingly was enriched for both embryo proper and suspensor transcripts. Most of the nuclei in clusters 3 and 12 were, respectively, labeled as columella initials and quiescent center initials, which are situated between the suspensor and embryo proper. Whereas cluster 3 was only enriched for suspensor transcripts, cluster 12 was enriched for both suspensor and embryo proper transcripts. We also confirmed these results with another published transcriptome dataset generated from embryo propers and suspensors of globular embryos (Zhou et al., 2020) (Fig. 2D). As further support for the cell type assignments of the clusters, three genes not included in our reference list were recently found to be specifically expressed in vascular initials (Smit et al., 2020) and all three were specific to cluster 11 (Fig. S3C) along with other vascular-expressed genes (Fig. 2C).

We then used RNA in situ hybridization (ISH) to further evaluate the marker-based assignments of snRNA-seq clusters to individual cell types. We selected 33 genes without reported expression patterns that represented a specific cell or group of cells based on their expression patterns (Fig. 2E; Fig. S3D). We could detect RNA ISH signal in at least $50 \%$ of embryos for 26 of the 33 probes tested (78.8\%) and compared the RNA ISH and snRNA-seq expression patterns for these in more detail (Fig. 2F; Fig. S3E,F and Fig. S4). AT3G13690, AT4G29020 and SERINE CARBOXYPEPTIDASELIKE 25 (SCPL25) were expressed at high levels in clusters 2, 5 and 6 , and detected by RNA ISH almost exclusively in the protoderm. AT5G01870 was also highly expressed in clusters 2, 5 and 6, as well as cluster 12 , and was detected in the protoderm and columella initials; whereas $A T 1 G 04880$ was expressed in clusters 6 and 9, and detected in the upper protoderm. AT1G80133, AT2G42660, AT3G54780 and GH3.2 were highly expressed in clusters 3 and 12 , and RNA ISH signals were detected in the columella and quiescent center initials, as well as throughout the suspensors for GH3.2. LITTLE ZIPPER 4 (ZPR4) was specifically expressed in cluster 7 based on snRNA-seq and detected in the shoot meristem initials by RNA ISH. RESPONSIVE TO DEHYDRATION 19 (RD19) transcripts were also detected by RNA ISH in shoot meristem initials, but were moderately expressed in all clusters. Similarly, AT4G38370 was expressed throughout the clusters, albeit most strongly in cluster 8 , but the RNA ISH signal was stronger in the embryo proper. These two apparent discrepancies between gene expression and RNA localization may be due to differences between nuclear and cytoplasmic mRNA populations, including variability in post-transcriptional regulation among cell types. ALCATRAZINTERACTING PROTEIN 1 (ACI1), AT3G15680 and AT3G15720 were expressed most highly in cluster 11 and detected in vascular initials with RNA ISH. ROOT MERISTEM GROWTH FACTOR 8 ( $R G F 8$ ) was highly expressed in clusters 11 and 12, and RGF8 transcripts were detected in vascular and columella initials. AT5G61412, BETA GLUCOSIDASE 17 (BGLU17), COBRALIKE PROTEIN 6 PRECURSOR (COBLO), CYSTEINE ENDOPEPTIDASE 1 (CEP1), EARLY NODULIN-LIKE PROTEIN 2 (ENODL2), MAJOR LATEX PROTEIN 28 (MLP28) and SPERMIDINE DISINAPOYL ACYLTRANSFERASE (SDT) were expressed in clusters 8,10 or 13 , and all their corresponding transcripts were detected in suspensors by RNA ISH. AT3G13230, MITOTIC ARREST-DEFICIENT 2 (MAD2) and TARGETING 
PROTEIN FOR XKLP2 (TPX2) were highly expressed in clusters 1, 5 and/or 9 , and corresponding RNA ISH produced 'salt-and-pepper' patterns, which are indicative of cell-cycle regulated genes. Accordingly, we observed that clusters 1,5 and 9 were enriched for mitotic-phase-regulated transcripts (Menges et al., 2003) (Fig. S3G). Genes preferentially expressed in clusters 1,5 and 9 also tended to be localized to the subprotoderm, protoderm or both layers, respectively. Therefore, our results suggested that clusters 1 , 5 and 9 represent dividing subprotoderm (dividing inner; div.i), protoderm (dividing outer; div.o) and dividing cells in general (div), respectively (Fig. 2G). Altogether, our in silico and in situ validations indicated that we can assign groups of snRNA-seq libraries to the major cell types present in globular embryos: the suspensor (sus1, cluster 10; sus2, cluster 8; sus3, cluster 13); columella initials (col; cluster 3), quiescent center initials (qc; cluster 12); vascular initials (vas; cluster 11); shoot meristem initials (smi; cluster 7); and the lower and upper protoderm (lpd, cluster 2; upd, cluster 6) (Fig. 2G).

\section{General characteristics of transcriptomes from embryonic cell types}

To provide a concise and uniform parameter to examine gene expression patterns across embryonic cell types, we calculated 'enrichment scores' in each of the 12 clusters for the 13,893 transcripts detected in $\geq 10 \%$ of nuclei within $\geq 1$ cluster (Table S3). Enrichment scores are a combination of the deviations of mean transcript levels and the percentage of nuclei it was detected in for each cluster relative to the other 11 clusters (see Materials and Methods; Table S3), and thus concisely summarize the relative abundance of each transcript in each cluster. The 250 genes with the highest enrichment scores (top-ranked 250) from each cluster were considered preferentially expressed genes for that cluster. Enrichment scores of known markers matched their reported expression patterns (Fig. 2C; Fig. S3D). For example, 74 of the $118(62.7 \%)$ reference genes were within top-ranked 250 genes of at least one cluster, including four that were top-ranked: $P I N$ FORMED 1 (PIN1; cluster 1), KANADI 1 (KAN1; cluster 2), WOX5 (cluster 12) and WUSCHEL RELATED HOMEOBOX 8 (WOX8; cluster 13). To gain insights into which biological processes are enriched in each embryonic cell type, we conducted gene ontology (GO) term enrichment analyses on the top-250 ranked genes of each cluster (Fig. 3A; Table S4). Significantly enriched GO terms were identified for the top-ranked 250 genes in the div, vas, div.i, smi, lpd, div.o and upd clusters, but not sus $1 / 2 / 3$, col or qc clusters. The inability to detect enriched terms in these BCL clusters may have been due to the limited annotation of genes specifically expressed in these cell types. Consistent with the div, div.i and div.o clusters representing actively dividing cells, GO terms related to progression through mitotic phases (div and div.o) and microtubules (div.i and div.o) were enriched. GO terms related to body axis specification were also enriched in the top-250 ranked genes of the div.i cluster, as well as the vas cluster. The 'microsporocyte differentiation' GO term is only associated with BARELY ANY MERISTEM 1 (BAM1) and BAM2, which encode receptor-like kinases. Both were among the top-250 ranked genes of the vas cluster (Table S3) and are required for vascular patterning in leaf and root tissues (DeYoung et al., 2006; Fan et al., 2021). The protoderm clusters (lpd and upd) were both enriched for specification of axis polarity and cutin biosynthesis terms within their top-250 ranked genes. Moreover, the top-250 ranked genes of the lpd and upd clusters could be distinguished from each other by their overrepresentation of epidermal and cotyledon development GO terms, respectively. The top- 250 ranked genes of the smi cluster were enriched for genes involved in DNA replication processes, including pre-replicative complex assembly, which is consistent with the smi cluster being depleted for mitosis phase markers (Fig. S3G). Overall, the enriched GO terms were consistent with the assigned cluster identities (Fig. 2G) and indicate that we have classified embryonic cell types with distinct functions.

Next, we tested whether genes essential for embryogenesis are preferentially enriched within the top- 250 ranked genes of each cluster. EMBRYO-DEFECTIVE (EMB) genes are a set of genes required for normal embryo development in Arabidopsis (Meinke, 2020). EMB genes were enriched in the top- 250 genes of the ACL clusters including significant enrichment in the smi, div.o and upd clusters. By contrast, EMB genes were depleted from top- 250 genes of the BCL clusters, including significant depletion in the col and sus2 clusters (Fig. 3B). Further supporting that genes preferentially expressed in the ACL are more likely to be required for proper development than those in the BCL, we found that the top- 250 ranked genes within the ACL, and especially the div, vas and lpd clusters, were more highly conserved across Brassicaceae species and land plants in general compared with BCL clusters (Haudry et al., 2013; Tian et al., 2020) (Fig. 3C; Fig. S5A,B). Also consistent with the EMB analyses, the top-250 ranked genes within the BCL clusters were more poorly conserved, especially genes enriched in the sus1, sus2, col and qc clusters. Altogether, these results suggested that genes preferentially expressed in ACL clusters, and especially the vas and div clusters, are under stronger purifying selection compared with those in BCL clusters, especially the col cluster, which are mutating at a faster rate. This is also consistent with the more variable morphologies of suspensors relative to embryo propers (Chen et al., 2021).

\section{Transcripts encoding epigenetic regulators vary across embryonic cell types}

Soon after fertilization of egg and sperm, epigenetic states are reprogrammed in the new generation (Gehring, 2019). This includes replacement of histones, as well as re-establishment of DNA methylation landscapes genome-wide by small RNA-dependent and -independent pathways (Bouyer et al., 2017; Ingouff et al., 2010; Jullien et al., 2012; Nagasaki et al., 2007; Papareddy et al., 2020). Because such differential chromatin states can strongly influence gene expression, we examined the transcript levels of genes previously implicated in chromatin regulation. More specifically, we found that 50/191 genes involved in general chromatin features, histone modifications (i.e. acetylation, methylation and ubiquitination), polycomb repressive complexes, DNA methylation or demethylation, or small RNA production or activities, had enrichment scores $\geq 2.5$ in $\geq 1$ embryonic cell cluster (Erdmann and Picard, 2020; Pikaard and Mittelsten Scheid, 2014) (Fig. 4A). General chromatin factors and components of the polycomb repressive complex tended to vary between the embryo proper and suspensor. HISTONE ACETYLTRANSFERASE OF THE CBP FAMILY 1 (HAC1) was enriched in the suspensor clusters, whereas HISTONE DEACETYLASE 3/4 (HDA3/4) were enriched in the embryo proper. Moreover, the JUMONJI DOMAIN-CONTAINING16/27/29 (JMJ16/27/29) and JMJ22 histone demethylases were enriched in the suspensor and embryo proper, respectively. Interestingly, the terminally differentiated suspensor was enriched for transcripts encoding proteins required for the production of 24-nt small interfering RNAs (siRNAs) such as CLASSY1 (CLSY1), NUCLEAR RNA POLYMERASE 
A

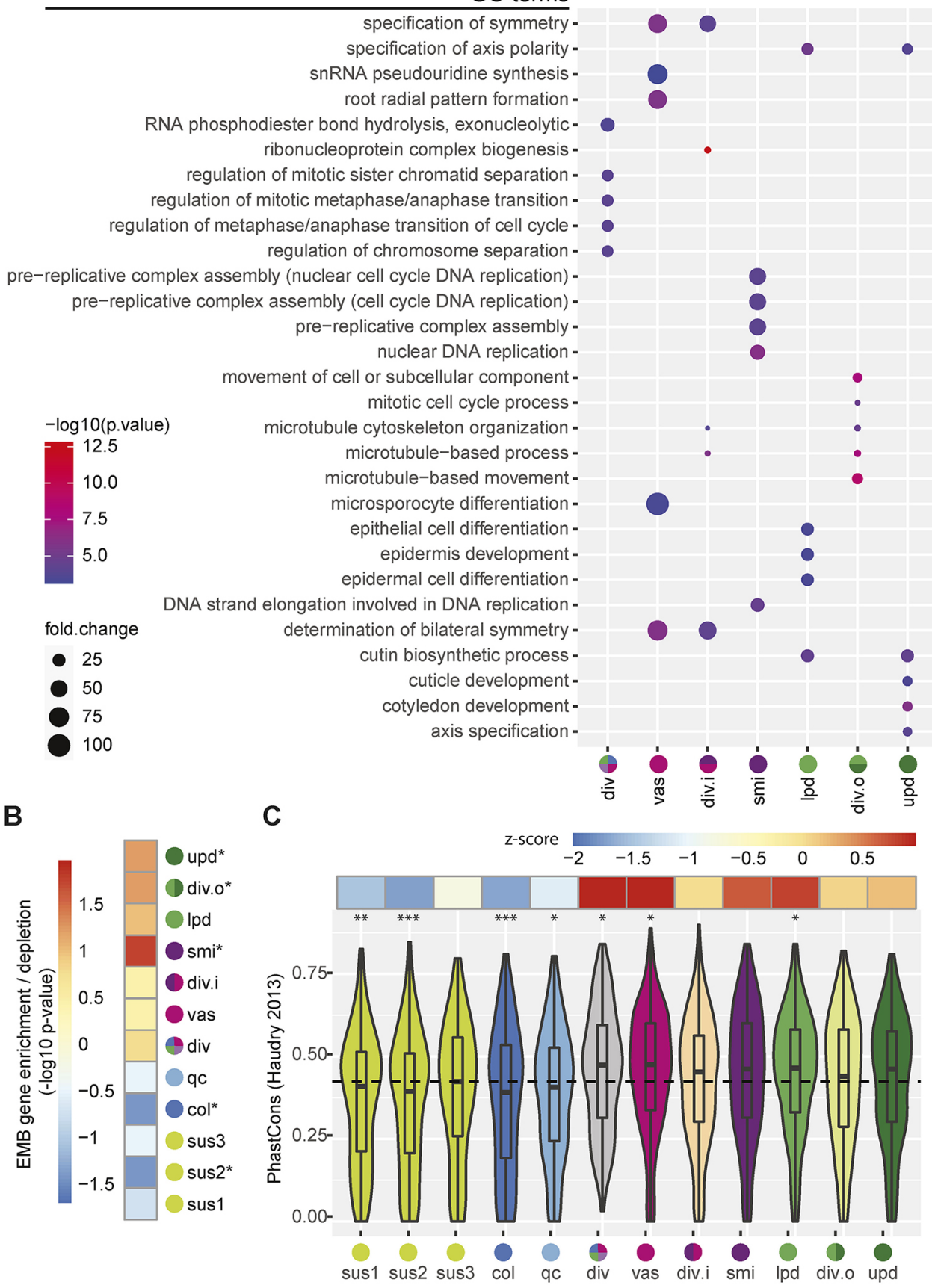

Fig. 3. General characteristics of transcriptomes from embryonic cell types. (A) The top-five enriched gene ontology (GO) terms identified by PANTHER for each cluster according to the top-250 ranked genes for each cluster. The suspensor clusters $(8,10,13)$ and hypophysis clusters $(3,12)$ did not have significantly enriched $\mathrm{GO}$ terms and thus are not shown. The sizes and colors of the dots represent the fold changes and $-\log _{10^{-}}$ transformed $P$-values, respectively. (B) Levels of overrepresentation of embryo-defective (EMB) genes for the top-250 ranked genes for each cluster. Asterisks indicate significant $(P \leq 0.05)$ enrichment or depletion of EMB genes relative to expectations. (C) PhastCons conservation scores (Haudry et al., 2013) of top-250 ranked genes for each cluster. The mean PhastCons score of all expressed genes is indicated by a dashed line, and deviations from the mean are presented in the upper row as $z$-scores. ${ }^{*} P \leq 0.05$, ${ }^{* *} P \leq 0.01,{ }^{* *} P \leq 0.001$; based on two-sided Kolmogorov-Smirnov tests with the alternative hypothesis that the cluster conservation score distributions of the top-ranked 250 genes were not equal to that of all expressed genes in embryos. PhastCons and PhyloP scores from another report (Tian et al., 2020) had similar trends (Fig. S5).
D1A (NRPD1A) and DICER-LIKE3 (DCL3) and this was consistent with previously published datasets (Belmonte et al., 2013; Zhou et al., 2020). By contrast, genes encoding Argonaute (AGO; AGO1/5/8/9/10) proteins, which bind to small RNAs and mediate gene repression, were enriched in the precursors of the shoot meristem initials. The enrichment of AGOs in shoot meristem initials is supported by previous reports (Gutzat et al., 2020; Jullien et al., 2020 preprint; Tucker et al., 2008) and is consistent with small RNA-mediated surveillance pathways that prevent transposon mobilization and other genome de-stabilizing events being enriched in the precursors to all aerial tissues including the gametes. Altogether, these results suggest that small RNAdependent and -independent pathways establish distinct chromatin environments in individual cell lineage precursors.
The most striking cell-specific enrichments were in pathways affecting cytosine methylation, which is typically associated with transcriptional silencing of transposons and repression of gene promoters (Law and Jacobsen, 2010). CHROMOMETHYLTRANSFERASE 3 (CMT3) and METHYLTRANSFERASE 1 (MET1) encode DNA methyltransferases that maintain cytosine methylation in the $\mathrm{CHG}(\mathrm{H} \neq \mathrm{G})$ and $\mathrm{CG}$ contexts, respectively, and both were enriched in the embryo proper. By contrast, transcripts encoding the REPRESSOR OF SILENCING (ROS1), DEMETERLIKE 2 (DML2) and DML3 DNA glycosylases required for the removal of methylated cytosines were highly enriched in the BCL including the suspensor, columella and quiescent center initials. Recently, 275 genes were found to be hypermethylated and downregulated in $\operatorname{ros} 1 \mathrm{dml} d \mathrm{dml} 3$ triple mutant $(r d d)$ seedlings 
A

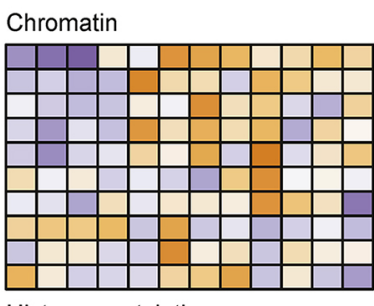

Histone acetylation

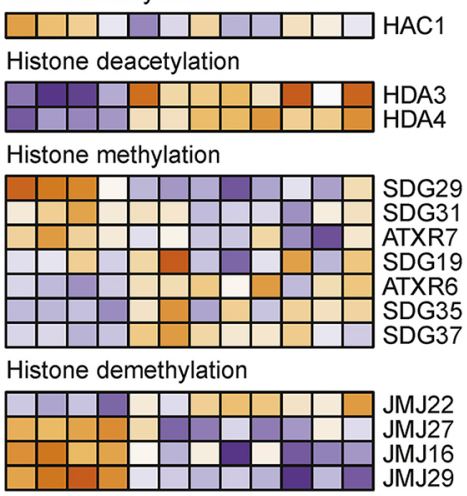

Histone ubiquitin

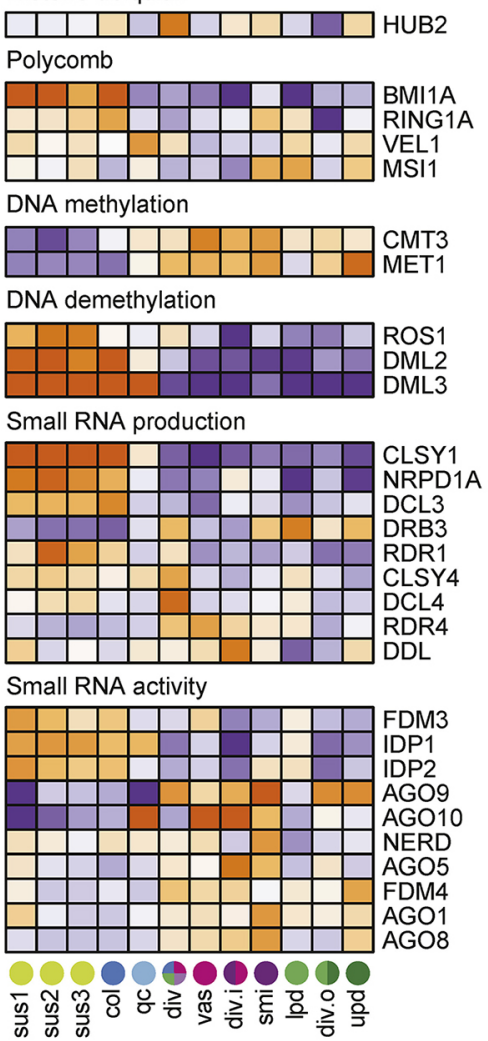
NRP2 FAS1 NAP1;1 SWP73B INO80 SYD CHR11 HAC1 DA3 S29 SDG31 DG19 ATXR6 SDG35 MJ27 MJ16 MJ29

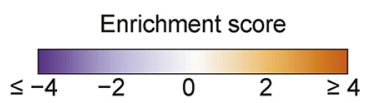

B Histone demethylation

\section{C}

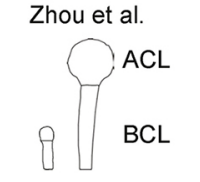

1-cell 32-cell

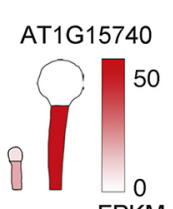

FPKM

AT3G26100
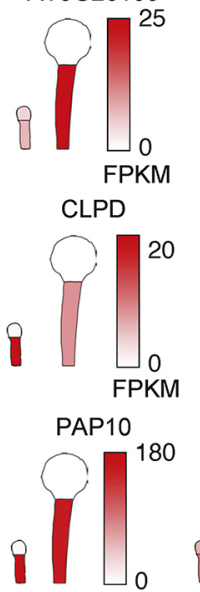

FPKM

FMO GS-OX3

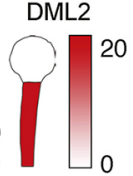

FPKM
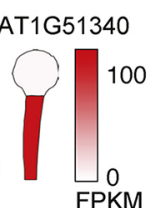

AT4G19860

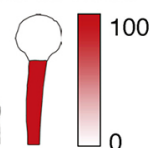

FPKM
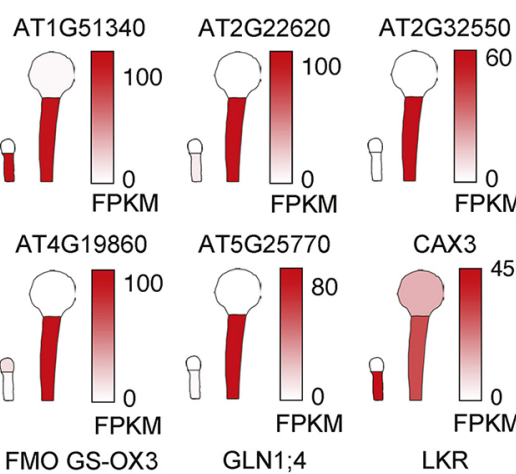

AT2G32550

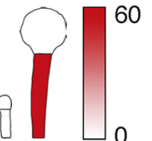

FPKM

AT5G25770

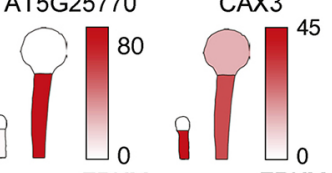

FPKM

GLN1;4

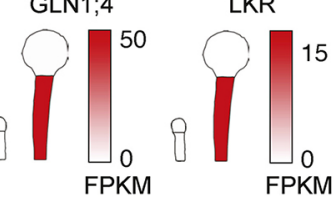

SBT3.12

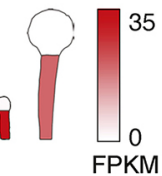

Fig. 4. Transcripts encoding epigenetic regulators vary across embryonic cell types. (A) Heatmap illustrating enrichment scores in 12 clusters corresponding to different embryonic cell types. Transcripts with enrichment scores $\geq 2.5$ in $\geq 1$ cell cluster are shown and enrichment scores are colored according to key. Gene names are indicated and cluster identities are marked and color-coded at the bottom according to Fig. 2G. (B) Violin plot (top) and heatmap (bottom) of enrichment scores for 16/50 ROS1/DML2/DML3 (RDD) targets detected and enriched in the basal cell lineage. (C,D) Schematic of RDD transcripts $(C)$ and their putative embryonic targets (D) based on published mRNA-seq from apical and basal cell lineages in one-cell and 32-cell stage embryos (Zhou et al., 2020).

Transcript levels (fragments per kilobase of transcript per million mapped reads; FPKM) are colored according to the keys. undergoing tracheary element differentiation and were considered to be a subset of direct ROS1/DML2/DML3 targets (i.e. RDD targets) (Lin et al., 2020). We detected 50/275 RDD targets in $\geq 10 \%$ of nuclei in $\geq 1$ embryonic cell cluster with enrichment scores $\geq 2$ (Fig. 4B; Fig. S6). Sixteen of these RDD targets were highly enriched in the BCL. ROS1, DML2 and DML3 transcripts were increased specifically in the BCL between the one-cell and 32-cell stages (Zhou et al., 2020) (Fig. 4C). Consistently, most embryonic RDD target candidates were also increased in the BCL during these early embryonic stages (Fig. 4D). Although we could not detect morphological defects in $r d d$ mutant embryos (Fig. S7), this may be due to redundancy with DEMETER (DME), which encodes a closely related DNA glycosylase family member (Choi et al., 2002; Gong et al., 2002). Consistent with this hypothesis, DME transcripts were enriched in the BCL and had increased levels in the BCL between the one-cell and 32-cell stages, similar to what we observed for ROS1, DML2 and DML3 (Fig. S7). Based on these results, we propose that DNA demethylases become activated in the BCL by the globular stage and catalyze the removal of methyl groups from a set of gene promoters to derepress their expression. 


\section{Differential enrichment of TF binding motifs}

To gain insights into the transcriptional processes that help define these embryonic cell-specific transcriptomes, we tested whether any consensus DNA motifs from the CIS-BP database of TF binding experiments (Weirauch et al., 2014) were overrepresented in the promoters of the top- 250 ranked genes of each cluster. We found that a total of $18 \mathrm{TF}$ motif families were overrepresented in at least one cluster (Fig. 5A). Overrepresentation of a motif suggests that at least one of the TF family members influences the expression of the top-250 preferentially expressed genes of that cluster. Families of TFs that bind nearly identical motifs can be very large, making it difficult to determine which TF or TFs in a family could be interacting with a given motif. We sought to generate a collection of candidate genes most likely to be interacting with each significant motif in the embryo. We considered a TF a candidate if its binding motif exists in the CIS-BP database and was enriched, or if an enriched motif exists in the database for a TF in the same subfamily. We examined the correlations between TF family motif enrichments and the expression enrichments of individual $\mathrm{TF}$ candidates (Table S5; Fig. 5B) and highlighted the candidate that was most strongly positively or negatively correlated (Fig. 5C). For example, WRKY DNA-BINDING PROTEIN 2 (WRKY2) is a transcriptional activator in the BCL and was shown to directly activate $W O X 8$ and $W O X 9$ (Ueda et al., 2011). Consistent with this report, the most overrepresented motif in BCL clusters was the Wbox bound by WRKY TFs, and this correlated well with the expression enrichment of WRKY2 (Pearson's $r=0.86$; Table S5). In addition to $W R K Y 2$, the expression pattern of two other WRKY TFs (WRKY28 and WRKY19) strongly correlated with enrichment of the WRKY motif (Pearson's $r=0.94$ and 0.96 , respectively; Fig. 5B,C).
The WOX family binding motif was similarly concentrated in BCL clusters, matching the observed expression pattern of $W O X 8$ (Fig. 5C) and to a lesser extent WOX9. The RNA encoding the B3 domain TF FUSCA3 (FUS3) is preferentially enriched in the $\mathrm{BCL}$, and the RY motif bound by FUS3 is similarly enriched only in $\mathrm{BCL}$ clusters. Maintenance of quiescent center (QC) identity in roots requires $J A C K D A W \quad(J K D)$, a member of the INDETERMINATE DOMAIN (IDD) subfamily of C2H2 zincfinger TFs (Welch et al., 2007). The IDD motif is enriched exclusively in the QC initials, in which $J K D$ is the second highest ranked gene behind WOX5. Class IV HOMEODOMAINLEUCINE ZIPPER (HD-ZIPs) include the L1 layer marker genes MERISTEM LAYER 1 (ATML1) and PROTODERMAL FACTOR 2 $(P D F 2)$, and their binding sites are overrepresented in the three protoderm clusters. The binding motifs of R1R2R3 Myb TFs, also known as mitosis-specific activator (MSA) elements, are enriched in the three clusters previously identified as actively dividing tissues (div, div.i, div.o), consistent with the role of R1R2R3 Myb TFs in positively regulating genes required for cytokinesis (Haga et al., 2007). Overall, the patterns of TF binding site enrichment are consistent with the literature on early embryo development, and the list of candidate TFs could serve as a valuable resource for future studies.

\section{DISCUSSION}

We developed a method to generate high-quality transcriptomes from single embryonic nuclei without detectable contamination from surrounding seed tissues (Fig. 1). Individual nuclear transcriptomes were then grouped according to their cell type, which were validated using published datasets and RNA ISH (Fig. 2). This allowed us to construct a gene expression atlas of Arabidopsis embryos at the

A

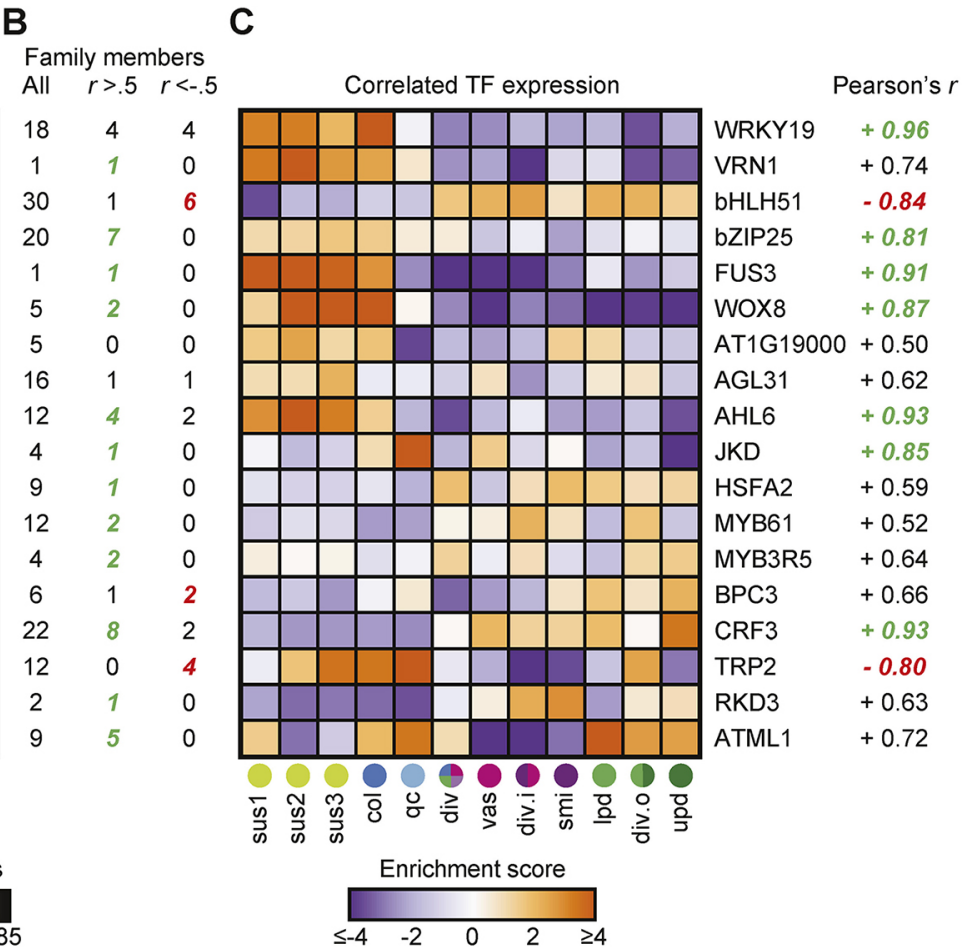

Fig. 5. Cluster-enriched TF binding motifs. (A) Dot plot of TF families with DNA binding motifs significantly enriched in at least one cluster. Dot size shows the most significant enrichment (- $\log _{10} P$-value, AME) of a motif in the family; dot color depicts the percentage of the top-250 ranked genes with a promoter containing

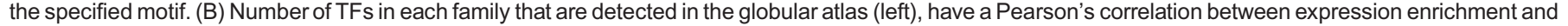
motif enrichment across clusters greater than 0.5 (center), or less than -0.5 (right). (C) Heatmap of expression enrichment scores for the TF within each family with an expression enrichment that correlates most strongly to motif enrichment. 
globular stage when the basic body plan is being established. Our results build upon foundational research examining the divergence of gene expression between the first two sporophytic cell lineages (Belmonte et al., 2013; Chen et al., 2021; Zhou et al., 2020) to help characterize how distinct gene expression programs, and corresponding cell types, are generated during early embryogenesis. Because evolutionary trajectories and transcripts encoding epigenetic factors or transcriptional regulators varied across early embryonic cell types, we surveyed these aspects to gain insights into how distinct gene expression programs are established in early embryos.

Consistent with a recent study, we found that genes preferentially expressed in suspensors tend to diverge more between species compared with those in embryo propers (Geist et al., 2019). Moreover, genes with enriched expression in columella initials were among the most rapidly evolving in the cell types we examined (Fig. 3C; Fig. S5). Conflict among siblings for maternal resources is thought to drive adaptive evolution of suspensors (Geist et al., 2019), which support the developing embryo proper and can serve as a conduit for maternally derived molecules (Nagl, 1990; Robert et al., 2018; Shi et al., 2019; Stadler et al., 2005; Yeung, 1980). Because columella initials are situated between the suspensor and embryo proper, they may help regulate communication between mothers and their offspring. In addition, DNA glycosylases required for demethylation of DNA are upregulated in the BCL of preglobular embryos, and our results are consistent with them catalyzing the removal of transcriptionally repressive methylation from gene promoters by the globular stage (Fig. 4). Interestingly, genes required for 24-nt siRNA biogenesis (e.g. CLSY1, NRPDIA and $D C L 3)$ were preferentially expressed in suspensors whereas transcripts encoding several AGO proteins that bind to small RNAs and mediate gene repression were enriched in embryo propers, especially shoot meristem initials from which the gametes are ultimately derived (Fig. 4A). Moreover, small RNAs can move between cells through plasmodesmata (Vatén et al., 2011), which also connect suspensors with embryo propers (Mansfield and Briarty, 1991). Similar to what has been proposed in other terminally differentiated cell-types in reproductive tissues (Calarco et al., 2012; Feng et al., 2013; Hsieh et al., 2009; Ibarra et al., 2012; Mosher and Melnyk, 2010; Slotkin et al., 2009), it is conceivable that suspensors generate large amounts of 24-nt siRNAs that flow into embryo propers and help silence and immobilize transposons to limit their mutagenic potential. Although beyond the scope of the current study, future cell-specific profiling of siRNAs and epigenetic marks in embryos should enable characterization of DNA demethylation and siRNA production in suspensors.

It is well-established that TFs drive pattern formation during animal embryogenesis, but relatively little is known about transcriptional regulation in plant embryos, partially due to redundancy among TF family members. By examining the relationships between $\mathrm{TF}$ expression levels and the enrichments/depletions of their corresponding binding motifs across embryonic cell types, we both verify existing models and provide testable hypotheses for how specific TFs influence cell-specific gene expression programs in early Arabidopsis embryos (Fig. 5; Table S5). For example, we observed characteristic patterns of TF binding motif enrichments and expression patterns in suspensors, quiescent center initials, subprotoderm, protoderm and shoot meristem initials (Fig. 5). WRKY2 regulates suspensor development by transcriptionally activating WOX 8 and WOX9, which in turn are redundantly required for suspensor development (Breuninger et al., 2008; Ueda et al., 2011, 2017). Accordingly, suspensors were enriched for WRKY and WOX motifs, as well as motifs for FUS3 which has also been implicated in suspensor development (Lotan et al., 1998). Although VRN and AHL TFs do not have reported functions in suspensors, their binding motifs and expression of their corresponding family members (i.e. VRN1, AHL1 and AHLO) were suspensor-enriched, which is consistent with transcriptional regulatory functions. TELOMERE BINDING PROTEIN (TBP) TFs have also not been implicated in suspensor development, and TBP expression and TBP binding motifs were enriched and depleted in suspensor-enriched gene promoters, respectively. TBPs can recruit polycomb group complexes (PcGs) to target loci and help repress their expression (Zhou et al., 2016, 2018). Similarly, BASIC PENTACYSTEINE (BPC) TFs can also recruit PcGs to target loci (Hecker et al., 2015; Xiao et al., 2017), and expression of specific BPC family members (e.g. BPC5/7) was enriched in suspensors, and BPC binding motifs were depleted from the promoters of top-250 ranked suspensor genes (Table S5). Future experiments are required to test whether TBP/BPC-mediated recruitment of $\mathrm{PcGs}$ and resulting epigenetic silencing is required for suspensor development.

The QC initials are derived from the uppermost derivative of the BCL and, unlike suspensors, contribute to post-embryonic tissues. IDD TF binding motifs were specifically enriched in the QC initials and expression of the IDD family member, JKD, which is required for QC identity in roots (Welch et al., 2007), was highly enriched in QC initials but not suspensors (Fig. 5). This implies that the superimposition of JKD on the BCL TF combinations could promote QC initial identity in early embryos. In contrast to the BCL, the subprotoderm (i.e. inner cells of the embryo proper) is enriched in motifs including those for BPC, TBP and ERF TFs (Fig. 5). Among several other ERF family members preferentially expressed in the embryo proper, DRN functions upstream of auxin and binds GCC motifs to promote meristem identity (Chandler et al., 2007; Eklund et al., 2011; Iwase et al., 2017; Kirch et al., 2003). The protoderm already expresses genes characteristic of specific processes inherent to the outermost layer during early embryogenesis (Fig. 3A) and is enriched for HD-ZIP IV TF motifs (Fig. 5A). Accordingly, transcripts encoding ATML1 and PDF2 family members were enriched in the protoderm and are required for its specification (Abe et al., 2003; Ogawa et al., 2015). Another cell-specific enrichment of cis-regulatory motifs was observed for RKD TFs in the shoot meristem initials. RKD genes tend to be expressed in reproductive tissues of land plants (Jeong et al., 2011; Koi et al., 2016; Kőszegi et al., 2011; Waki et al., 2011) and their overexpression is sufficient to induce expression of undifferentiated cell types (Köszegi et al., 2011; Waki et al., 2011). Therefore, the enrichment of RKD motifs, as well as the preferential expression of $R K D 3 / 5$ family members, in the shoot meristem initials make $R K D 3 / 5$ good candidates for future investigation into the establishment of shoot meristem initial gene expression programs.

In addition to providing an early embryonic gene expression atlas, the presented workflow may help guide snRNA-seq experiments on embryos and other plant tissues that are difficult to access. The future application of similar techniques across embryonic stages in Arabidopsis and other species should contribute to a deeper understanding of how gene expression programs are dynamically established during plant embryogenesis. Moreover, integrating snRNA-seq data with other single-cell genomic technologies such as single-cell ATAC-seq (Buenrostro et al., 2015; Cusanovich et al., 2015) may allow further characterization of gene regulatory mechanisms operating in plant embryos. We expect that, together with more focused studies, these genome-wide datasets will accelerate our understanding of the molecular basis of pattern formation in plant embryos. 


\section{MATERIALS AND METHODS}

Plant materials, growth conditions and microscopy

Arabidopsis thaliana accession Columbia (Col-0) plants containing pWOX2::H2B-GFP, pWOX2::tdTomato-RCI2b (pWOX2::NLS-GFP) (Gooh et al., 2015) or no transgenes were grown at $20-22^{\circ} \mathrm{C}$ and $16 \mathrm{~h}$ light $/ 8 \mathrm{~h}$ dark cycles under incandescent lights $\left(130-150 \mu \mathrm{mol} / \mathrm{m}^{2} / \mathrm{s}\right)$ in a climate-controlled growth chamber. The $r d d$ triple mutants were composed of ros 1-3, dml2-1 and $d m l 3-1$ (Penterman et al., 2007) and Nomarski microscopy was carried out as previously described (Plotnikova et al. 2019).

\section{Nuclei isolation and FANS}

Developing seeds containing globular embryos from the transgenic pWOX2::H2B-GFP, pWOX2::tdTomato-RCI2b lines and wild-type Col-0 were isolated before sorting. For each set, developing seeds were isolated with tungsten needles under a stereomicroscope from 20 self-pollinated siliques at stage 17 (Smyth et al., 1990), corresponding to $72 \mathrm{~h}$ after pollination when most embryos are at the early/mid-globular stage under the growth conditions used. Developing seeds were isolated at the same time of day to minimize variations caused by circadian rhythms and immediately transferred to $600 \mu \mathrm{l}$ cooled fixative buffer consisting of $1 \times$ Galbraith's buffer [20 mM MOPS ( $\mathrm{pH} 7.0$ ), $30 \mathrm{mM}$ sodium citrate, 1\% Triton X-100, $45 \mathrm{mM} \mathrm{MgCl}_{2}$ ] and $500 \mu \mathrm{M}$ dithiobis(succinimidyl propionate) (DSP; Thermo Fisher Scientific). All buffers used in the nuclei isolation and sorting contained $0.4 \mathrm{U} / \mathrm{ml}$ RNAse inhibitor murine (New England Biolabs). Crosslinked samples were incubated with $800 \mu \mathrm{l}$ quenching buffer [1 $\mathrm{M}$ Tris-HCl ( $\mathrm{pH} 7.0), 30 \mathrm{mM}$ sodium citrate, $1 \%$ Triton $\mathrm{X}-100$ and $\left.45 \mathrm{mM} \mathrm{MgCl}_{2}\right]$ at room temperature for $15 \mathrm{~min}$ with gentle shaking. The quenched samples were washed twice with $600 \mu \mathrm{l} \mathrm{HG-GB}(1 \times$ Galbrath's buffer and $1 \mathrm{M}$ hexylene glycerol; Sigma-Aldrich). The seeds were then gently homogenized with micro-pestles in $1.5 \mathrm{ml}$ microtubes with $200 \mu \mathrm{l} \mathrm{HG}$ -

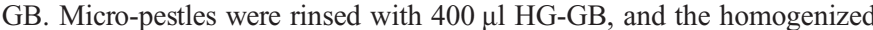
samples were gently pipetted ten times before incubating at $4^{\circ} \mathrm{C}$ for $15 \mathrm{~min}$ to maximize nuclei release. The partially homogenized samples were then filtered with $30 \mu \mathrm{m}$ filters and collected in $2 \mathrm{ml}$ microtubes. Another $600 \mu \mathrm{l}$ HG-GB were added to the $1.5 \mathrm{ml}$ microtube, and filtered and collected through the same $30 \mu \mathrm{m}$ filter and $2 \mathrm{ml}$ microtube, respectively, to maximize nuclei recovery. The filtered samples were then centrifuged at $1000 \mathrm{~g}$ at $4^{\circ} \mathrm{C}$ for $10 \mathrm{~min}$. The supernatant was carefully removed without disturbing the grayish pellet of nuclei. A fresh aliquot of $1 \mathrm{ml} \mathrm{HG}-\mathrm{GB}$ and $1 \mu \mathrm{l}$ of $10 \mathrm{mg} / \mathrm{ml}$ DAPI was added into microtubes and the pellet was gently re-suspended. Samples were then washed five times, including a 10 -min centrifugation at $1000 \mathrm{~g}$ at $4^{\circ} \mathrm{C}$ and replacement of supernatant with fresh aliquots of $1 \mathrm{ml} 1 \times$ Galbrath's buffer. The washed nuclei were then re-suspended in $800 \mu 11 \times$ Galbraith's buffer for sorting.

The isolated nuclei were sorted with a BD FACSAriaTM III Cell Sorter (BD Biosciences) with a $70 \mu \mathrm{m}$ nozzle. The scatter gates were adjusted accordingly with Col-0 nuclei. DAPI signals were activated by a $375 \mathrm{~nm}$ laser and collected with a 450/40 nm filter. GFP signals were activated by a $488 \mathrm{~nm}$ laser and collected with a $530 / 30 \mathrm{~nm}$ filter. To maximize purity, only the droplets containing a DAPI signal within the two peak regions representing 2 constant $(2 \mathrm{C})$ and 4 constant (4C) nuclei (Fig. S1A,C) were considered for GFP gating. For GFP gating, a region with low autofluorescence and high GFP signal was selected (Fig. S1B,D), which had less than three events in Col-0 samples and on average $\geq 200$ events for pWOX2::NLS-GFP samples. Each nucleus passing both DAPI and GFP gating was collected with single-cell settings in $4 \mu \mathrm{l}$ of cell lysis buffer (Picelli et al., 2014a) supplemented with 25 mM DTT in single wells of 96well plates.

\section{snRNA-seq}

Smart-seq2 libraries were prepared following the published SmartSeq2 single-cell protocol (Picelli et al., 2014a,b) with an additional 30-min $37^{\circ} \mathrm{C}$ incubation before reverse transcription. Libraries were sequenced on an Illumina Hi-Seq 2500 in 50-base single end mode. Sequencing reads from each sample were preprocessed by trimming adapters using cutadapt v2.6 (Martin, 2011) in two steps. First, Nextera adapters
(5'-CTGTCTCTTATACACATCTCCGAGCCCACGAGAC-3') were trimmed from the $3^{\prime}$ end of reads, followed by trimming of templateswitching oligos (TSO; 5'-AAGCAGTGGTATCAACGCAGAGTACATGGG-3') and oligo-dT adapters (5'-AAGCAGTGGTATCAACGCAGAGTACTTTTTTTTTTTTTTTTTTTTTTTTTTTTTT-3') from the $5^{\prime}$ and $3^{\prime}$ ends of reads, respectively. A Kallisto index was built from a combined FASTA file of all transcript models in EnsemblPlants TAIR10 v40 (ftp://ftp.ensemblgenomes.org/pub/plants/release-40/gff3/ arabidopsis_thaliana/Arabidopsis_thaliana.TAIR10.40.gff3.gz), 96 ERCC spike-in transcripts and sGFP. Each trimmed sample FASTQ file was pseudoaligned to this index using the command 'kallisto quant' with the options '-single -fragment-length $200-$ sd 100 ' to produce a table of transcripts per million (TPM) for each sample.

\section{Quality control and census count conversion}

The TPM table, cell data and gene data were imported into Monocle3 (Cao et al., 2019). Libraries with less than either 100,000 aligned reads or 1000 detected genes were considered as low quality and excluded from subsequent analyses. The TPM values were then converted to census counts with the census conversion algorithm (Qiu et al., 2017; Trapnell et al., 2014). The census counts were used as gene expression levels in the subsequent analyses.

\section{Maternal contamination removal and tissue enrichment tests}

Gene expression values were used to perform tissue enrichment tests with default settings as described (Schon and Nodine, 2017). The census count expression and metadata of snRNA-seq libraries from eight plates (Table S1) were constructed as a cell data set (CDS) in Monocle3 with R version 3.6.3. The quality control was carried out according to Monocle3 guidelines. Genes passing the Monocle3 function detect_genes(CDS, min_expr $=0.1$ ) and expressed in at least three nuclei were considered in subsequent analyses. The above quality control steps resulted in a CDS with 534 nuclei and 24,591 genes. An unsupervised UMAP (McInnes et al., 2018 preprint) dimension reduction and clustering performed on this CDS resulted in 20 clusters. Two of the clusters (Clusters 12 and 13 in Fig. S2) were dominated by nuclei that resembled the seed coat reference according to tissue enrichment tests, and therefore corresponding nuclei were excluded from subsequent analyses. After contamination removal, a CDS containing 486 globular embryonic nuclei and 23,959 detectable genes was then used for subsequent cell type score calculation and clustering.

\section{Calculation of cell type scores for globular nuclei and clustering}

A set of 174 embryonic marker genes based on either RNA ISH or transcriptional/translational fusions to fluorescent or beta-glucuronidase (GUS) reporters were collected from the literature (Table S2). Expression levels were recorded as strongly expressed (s), weakly expressed (w), not expressed (n) or non-informative (NA) for each of nine cell types: upd, lpd, smi, upper inner periphery (uip), vas, ground tissue initials (grd), qc, col and sus. The corresponding $174 \times 9$ matrix was intersected with expressed genes in our globular snRNA-seq libraries, which had at least one census count in at least seven nuclei. The resulting 135 expressed marker genes served as the reference for cell type-score calculations, with $56,52,38,43,62,43,56,51$ and 29 positive markers (i.e. strongly or weakly expressed) and 79, 83, 97, $92,73,92,79,84$ and 98 negative markers (i.e. not expressed) for upd, lpd, smi, uip, vas, grd, qc, col and sus, respectively. We used two-tailed hypergeometric tests assuming that a nucleus expressing positive and negative markers of a cell type was more or less likely to be from that cell type, respectively. The resulting $P$-values were $-\log _{10}$-transformed to compute cell type scores. The $486 \times 9$ matrix of cell type scores was then used for dimension reduction and clustering. Cluster identities were predicted based on the cell type labels within each cluster.

\section{Validation of cluster identities}

The mean expression values of all nuclei within each cluster were used to perform tissue enrichment tests as previously described (Schon and Nodine, 2017) and to calculate Spearman's correlation coefficients with published globular stage embryo proper (32E) and suspensor (32S) samples (Zhou 
et al., 2020). The expression levels of selected markers and three recently reported genes [PEAR1 (AT2G37590), DOF6 (AT3G45610) and GATA20 $(A T 2 G 18380)]$ (Smit et al., 2020) not included in our reference marker table for tissue score calculation were plotted with the Monocle3 'plot_genes_by_group()' function.

We selected 33 RNA ISH candidates without previously reported embryonic expression patterns according to their expression patterns and probe specificity (Table S6). RNA in situ probes were generated from synthesized double-stranded DNA (gBlocks Gene Fragments; Integrative DNA Technologies) and applied as previously described (Nodine et al., 2007). For each probe, 21-122 globular stage embryos (i.e. biological replicates) were examined from two to eight microscope slides (i.e. technical replicates) for a total of 1420 embryos from 112 slides (Fig. S4). To minimize potential bias, all in situ images were examined and classified by someone that did not perform the experiments and did not know the identities of the samples.

\section{Ranked gene enrichment}

For each cluster of nuclei, a ranked gene enrichment strategy was defined as follows: let $G$ be the set of 'expressed' genes, defined as all nuclear-encoded and RNA Polymerase II-transcribed genes with $\geq 1$ RNA-seq read count in $\geq 10 \%$ of nuclei in $\geq 1$ cluster. For each gene $i$ in each nucleus $j$, $C P M_{i j}=10^{6} \times$ counts $_{i j} / \sum_{g \in G}$ counts $_{g j}$. Let $C$ be a set of nuclei in a cluster and $|C|$ the number of nuclei in cluster $C$. Mean CPM of gene $i$ in cluster $C$ is defined as $\mu_{i C}=\sum_{j \in C} C P M_{i j} /|C|$. Proportion detected $p$ is defined for each gene $i$ in each cluster $C$ as the number of nuclei in which gene $i$ was detected: $p_{i C}=\left(\sum_{j \in C}\left\{\begin{array}{l}1 \text { if } \text { counts }_{i j} \geq 1 \\ 0 \text { if } \text { counts }_{i j}<1\end{array}\right) /|C|\right.$. Using one cluster $C$ as an ingroup and all other clusters as outgroup $O$, a mean CPM $\log _{2}$ fold change of each gene $i$ is calculated as $F_{i C}=\log _{2}\left(1+\mu_{i C} / 1+\sum_{o \in O} \mu_{i o} /|O|\right)$, and a mean proportion difference $D_{i C}=p_{i C}-\sum_{o \in O} p_{i o}$. Both sets $F_{C}$ and $D_{C}$ were centered and mean-scaled so that $\widehat{F_{C}}=F_{C}-\overline{F_{C}} / \sigma\left(F_{C}\right)$, and $\widehat{D_{C}}=D_{C}-\overline{D_{C}} / \sigma\left(D_{C}\right)$, where $\bar{x}$ is the mean and $\sigma(x)$ the standard deviation. Enrichment magnitude $E_{i C}$ of gene $i$ in cluster $C$ is the combined deviation from the mean of $F_{C}$ and $D_{C}$ :

$$
E_{i C}=\sqrt{{\widehat{F_{i C}}}^{2}+{\widehat{D_{i C}}}^{2}} \times\left\{\begin{array}{c}
1 \text { if } \widehat{F_{i C}}+\widehat{D_{i C}}>0 \\
-1 \text { if } \widehat{F_{i C}}+\widehat{D_{i C}} \leq 0
\end{array}\right.
$$

In each cluster, genes were ranked from highest to lowest enrichment magnitude and the first 250 genes and last 250 genes were considered 'topranked genes' and 'bottom-ranked genes', respectively (Table S3).

\section{Gene ontology analyses}

The IDs of the top-250 ranked genes for each cluster were submitted to TAIR GO Term enrichment (https://www.arabidopsis.org/tools/go_term enrichment.jsp) using the PANTHER classification system (Mi et al., 2021) to compute false discovery rates with Fisher's exact tests. All enriched terms are presented in Table S4. The five most significant GO terms not related to ribosomes are highlighted in Fig. 3.

\section{TF binding site analyses}

Promoters for all genes were defined as the region $500 \mathrm{bp}$ upstream to $100 \mathrm{bp}$ downstream of the most common $5^{\prime}$ end in nanoPARE datasets of globular-stage embryos (Plotnikova et al., 2019). For genes without nanoPARE signal, the most upstream $5^{\prime}$ end annotated in TAIR10 v.46 was used. TF binding motifs for Arabidopsis thaliana were downloaded from CIS-BP (http://cisbp.ccbr.utoronto.ca) (Weirauch et al., 2014). All directly determined motifs were tested for statistical overrepresentation using Analysis of Motif Enrichment (AME; http://meme-suite.org/doc/ame. html) (McLeay and Bailey, 2010) in each cluster by comparing the top250 ranked gene promoters against a background set of the bottom- 250 ranked gene promoters with default parameters. Motifs that were significantly enriched in at least one cluster were collapsed into motif families. The cluster-specific expression of all genes with a significantly enriched motif were tested for correlation with the cluster-specific pattern of motif family enrichment, as well as all genes not represented in the CIS-BP database, but in the same TF subfamily as a gene with a significant motif.

\section{Acknowledgements}

We are grateful to Daisuke Kurihara and Tetsuya Higashiyama for generously sharing pWOX2::H2B-GFP, pWOX2::tdTomato-RCI2b lines. Special thanks to the IMP-IMBA-GMI BioOptics Core Facility, the Plant Sciences Facility and the Next Generation Sequencing Facility at Vienna BioCenter Core Facilities $\mathrm{GmbH}$ (VBCF) for their technical support. We also thank Ortrun Mittelsten Scheid and Dolf Weijers for valuable comments on the manuscript. Ping Kao also personally thanks Kotoha Tanaka and Kaori Sakuramori for their support.

\section{Competing interests}

The authors declare no competing or financial interests.

\section{Author contributions}

Conceptualization: P.K., M.A.S., M.D.N.; Methodology: P.K., M.A.S.; Investigation P.K., M.A.S., M.M., B.E., M.D.N.; Writing - original draft: P.K., M.A.S., M.D.N.; Writing - review \& editing: P.K., M.A.S., M.D.N.; Visualization: P.K., M.A.S., B.E., M.D.N.; Supervision: M.D.N.; Funding acquisition: M.D.N.

\section{Funding}

This work was funded by Österreichischen Akademie der Wissenschaften Research infrastructures in Austria and Czech Republic (RIAT-CZ) project (ATCZ40) was funded via Interreg V-A Austria-Czech Republic, which is gratefully acknowledged for the financial support of the measurements for the preliminary snRNA-seq experiments at the Vienna Biocenter Core Facilities GmbH (VBCF) Next Generation Sequencing Facility. Open access funding provided by Wageningen University and Research Centre. Deposited in PMC for immediate release.

\section{Data availability}

All sequencing data generated in this study have been deposited in GEO under accession number GSE169284.

\section{Peer review history}

The peer review history is available online at https://journals.biologists.com/dev/ article-lookup/doi/10.1242/dev.199589

\section{References}

Abe, M., Katsumata, H., Komeda, Y. and Takahashi, T. (2003). Regulation of shoot epidermal cell differentiation by a pair of homeodomain proteins in Arabidopsis. Development 130, 635-643. doi:10.1242/dev.00292

Bai, S.-N. (2015). The concept of the sexual reproduction cycle and its evolutionary significance. Front. Plant Sci. 6, 11.

Belmonte, M. F., Kirkbride, R. C., Stone, S. L., Pelletier, J. M., Bui, A. Q., Yeung, E. C., Hashimoto, M., Fei, J., Harada, C. M., Munoz, M. D. et al. (2013). Comprehensive developmental profiles of gene activity in regions and subregions of the Arabidopsis seed. Proc. Natl. Acad. Sci. USA 110, E435-E444. doi:10.1073/ pnas.1222061110

Bouyer, D., Kramdi, A., Kassam, M., Heese, M., Schnittger, A., Roudier, F. and Colot, V. (2017). DNA methylation dynamics during early plant life. Genome Biol. 18, 179. doi:10.1186/s13059-017-1313-0

Bray, N. L., Pimentel, H., Melsted, P. and Pachter, L. (2016). Near-optimal probabilistic RNA-seq quantification. Nat. Biotechnol. 34, 525-527. doi:10.1038/ nbt.3519

Brennecke, P., Anders, S., Kim, J. K., Kołodziejczyk, A. A., Zhang, X., Proserpio, V., Baying, B., Benes, V., Teichmann, S. A., Marioni, J. C. et al. (2013). Accounting for technical noise in single-cell RNA-seq experiments. Nat. Methods 10, 1093-1095. doi:10.1038/nmeth.2645

Breuninger, H., Rikirsch, E., Hermann, M., Ueda, M. and Laux, T. (2008) Differential expression of WOX genes mediates apical-basal axis formation in the Arabidopsis embryo. Dev. Cell 14, 867-876. doi:10.1016/j.devcel.2008.03.008

Buenrostro, J. D., Wu, B., Litzenburger, U. M., Ruff, D., Gonzales, M. L., Snyder, M. P., Chang, H. Y. and Greenleaf, W. J. (2015). Single-cell chromatin accessibility reveals principles of regulatory variation. Nature 523, 486-490. doi:10.1038/nature14590

Calarco, J. P., Borges, F., Donoghue, M. T. A., Van Ex, F., Jullien, P. E., Lopes T., Gardner, R., Berger, F., Feijó, J. A., Becker, J. D. et al. (2012) Reprogramming of DNA methylation in pollen guides epigenetic inheritance via small RNA. Cell 151, 194-205. doi:10.1016/j.cell.2012.09.001

Cao, J., Spielmann, M., Qiu, X., Huang, X., Ibrahim, D. M., Hill, A. J., Zhang, F. Mundlos, S., Christiansen, L., Steemers, F. J. et al. (2019). The single-cell transcriptional landscape of mammalian organogenesis. Nature 566, 496-502. doi:10.1038/s41586-019-0969-x 
Casson, S., Spencer, M., Walker, K. and Lindsey, K. (2005). Laser capture microdissection for the analysis of gene expression during embryogenesis of Arabidopsis. Plant J. 42, 111-123. doi:10.1111/j.1365-313X.2005.02355.x

Chandler, J. W., Cole, M., Flier, A., Grewe, B. and Werr, W. (2007). The AP2 transcription factors DORNRÖSCHEN and DORNRÖSCHEN-LIKE redundantly control Arabidopsis embryo patterning via interaction with PHAVOLUTA. Development 134, 1653-1662. doi:10.1242/dev.001016

Chen, G., Ning, B. and Shi, T. (2019). Single-cell RNA-seq technologies and related computational data analysis. Front. Genet. 10, 317. doi:10.3389/fgene 2019.00317

Chen, M., Lin, J.-Y., Wu, X., Apuya, N. R., Henry, K. F., Le, B. H., Bui, A. Q., Pelletier, J. M., Cokus, S., Pellegrini, M. et al. (2021). Comparative analysis of embryo proper and suspensor transcriptomes in plant embryos with different morphologies. Proc. Natl. Acad. Sci. USA 118, e2024704118. doi:10.1073/pnas. 2024704118

Cheng, C.-Y., Krishnakumar, V., Chan, A. P., Thibaud-Nissen, F., Schobel, S. and Town, C. D. (2017). Araport11: a complete reannotation of the Arabidopsis thaliana reference genome. Plant J. 89, 789-804. doi:10.1111/tpj.13415

Choi, Y., Gehring, M., Johnson, L., Hannon, M., Harada, J. J., Goldberg, R. B. Jacobsen, S. E. and Fischer, R. L. (2002). DEMETER, a DNA glycosylase domain protein, is required for endosperm gene imprinting and seed viability in arabidopsis. Cell 110, 33-42. doi:10.1016/S0092-8674(02)00807-3

Clark, N. L., Aagaard, J. E. and Swanson, W. J. (2006). Evolution of reproductive proteins from animals and plants. Reproduction 131, 11-22. doi:10.1530/rep.1. 00357

Cusanovich, D. A., Daza, R., Adey, A., Pliner, H. A., Christiansen, L., Gunderson, K. L., Steemers, F. J., Trapnell, C. and Shendure, J. (2015) Multiplex single-cell profiling of chromatin accessibility by combinatorial cellular indexing. Science 348, 910-914. doi:10.1126/science.aab1601

DeYoung, B. J., Bickle, K. L., Schrage, K. J., Muskett, P., Patel, K. and Clark S. E. (2006). The CLAVATA1-related BAM1, BAM2 and BAM3 receptor kinaselike proteins are required for meristem function in Arabidopsis. Plant J. 45, 1-16 doi:10.1111/j.1365-313X.2005.02592.x

Dresselhaus, T. and Jürgens, G. (2021). Comparative embryogenesis in angiosperms: activation and patterning of embryonic cell lineages. Annu. Rev. Plant Biol. 72, 1-36. doi:10.1146/annurev-arplant-082520-094112

Efroni, I. and Birnbaum, K. D. (2016). The potential of single-cell profiling in plants. Genome Biol. 17, 65. doi:10.1186/s13059-016-0931-2

Eklund, D. M., Cierlik, I., Ståldal, V., Claes, A. R., Vestman, D., Chandler, J. and Sundberg, E. (2011). Expression of Arabidopsis SHORT INTERNODES/ STYLISH family genes in auxin biosynthesis zones of aerial organs is dependent on a GCC box-like regulatory element. Plant Physiol. 157 2069-2080. doi:10.1104/pp.111.182253

Erdmann, R. M. and Picard, C. L. (2020). RNA-directed DNA Methylation. PLoS Genet. 16, e1009034. doi:10.1371/journal.pgen.1009034

Fan, P., Aguilar, E., Bradai, M., Xue, H., Wang, H., Rosas-Diaz, T., Tang, W., Wolf, S., Zhang, H., Xu, L. et al. (2021). The receptor-like kinases BAM1 and BAM2 are required for root xylem patterning. Proc. Natl. Acad. Sci. USA 118, e2022547118. doi:10.1073/pnas.2022547118

Farmer, A., Thibivilliers, S., Ryu, K. H., Schiefelbein, J. and Libault, M. (2021) Single-nucleus RNA and ATAC sequencing reveals the impact of chromatin accessibility on gene expression in Arabidopsis roots at the single-cell level. Mol. Plant. 14, 372-383. doi:10.1016/j.molp.2021.01.001

Feng, X., Zilberman, D. and Dickinson, H. (2013). A conversation across generations: soma-germ cell crosstalk in plants. Dev. Cell 24, 215-225. doi:10. 1016/j.devcel.2013.01.014

Gehring, M. (2019). Epigenetic dynamics during flowering plant reproduction evidence for reprogramming? New Phytol. 224, 91-96. doi:10.1111/nph.15856

Geist, K. S., Strassmann, J. E. and Queller, D. C. (2019). Family quarrels in seeds and rapid adaptive evolution in Arabidopsis. Proc. Natl. Acad. Sci. USA 116 9463-9468. doi:10.1073/pnas.1817733116

Gerri, C., Menchero, S., Mahadevaiah, S. K., Turner, J. M. A. and Niakan, K. K. (2020). Human embryogenesis: a comparative perspective. Annu. Rev. Cell Dev. Biol. 36, 411-440. doi:10.1146/annurev-cellbio-022020-024900

Gong, Z., Morales-Ruiz, T., Ariza, R. R., Roldán-Arjona, T., David, L. and Zhu, J.K. (2002). ROS1, a repressor of transcriptional gene silencing in Arabidopsis encodes a DNA glycosylase/lyase. Cell 111, 803-814. doi:10.1016/S00928674(02)01133-9

Gooh, K., Ueda, M., Aruga, K., Park, J., Arata, H., Higashiyama, T. and Kurihara, D. (2015). Live-cell imaging and optical manipulation of Arabidopsis early embryogenesis. Dev. Cell 34, 242-251. doi:10.1016/j.devcel.2015.06.008

Gutzat, R., Rembart, K., Nussbaumer, T., Hofmann, F., Pisupati, R., Bradamante, G., Daubel, N., Gaidora, A., Lettner, N., Donà, M. et al. (2020). Arabidopsis shoot stem cells display dynamic transcription and DNA methylation patterns. EMBO J. 39, e103667. doi:10.15252/embj.2019103667

Habib, N., Li, Y., Heidenreich, M., Swiech, L., Avraham-Davidi, I., Trombetta, J. J., Hession, C., Zhang, F. and Regev, A. (2016). Div-seq: single-nucleus RNA-Seq reveals dynamics of rare adult newborn neurons. Science 353 925-928. doi:10.1126/science.aad7038
Haecker, A., Groß-Hardt, R., Geiges, B., Sarkar, A., Breuninger, H., Herrmann, M. and Laux, T. (2004). Expression dynamics of WOX genes mark cell fate decisions during early embryonic patterning in Arabidopsis thaliana. Development 131, 657-668. doi:10.1242/dev.00963

Haga, N., Kato, K., Murase, M., Araki, S., Kubo, M., Demura, T., Suzuki, K. Müller, I., Voß, U., Jürgens, G. et al. (2007). R1R2R3-Myb proteins positively regulate cytokinesis through activation of KNOLLE transcription in Arabidopsis thaliana. Development 134, 1101-1110. doi:10.1242/dev.02801

Haudry, A., Platts, A. E., Vello, E., Hoen, D. R., Leclercq, M., Williamson, R. J. Forczek, E., Joly-Lopez, Z., Steffen, J. G., Hazzouri, K. M. et al. (2013). An atlas of over 90,000 conserved noncoding sequences provides insight into crucifer regulatory regions. Nat. Genet. 45, 891-898. doi:10.1038/ng.2684

Hecker, A., Brand, L. H., Peter, S., Simoncello, N., Kilian, J., Harter, K., Gaudin V. and Wanke, D. (2015). The Arabidopsis GAGA-binding factor BASIC PENTACYSTEINE6 Recruits the POLYCOMB-REPRESSIVE COMPLEX1 component LIKE HETEROCHROMATIN PROTEIN1 to GAGA DNA Motifs. Plant Physiol. 168, 1013-1024. doi:10.1104/pp.15.00409

Hofmann, F., Schon, M. A. and Nodine, M. D. (2019). The embryonic transcriptome of Arabidopsis thaliana. Plant Reprod. 32, 77-91. doi:10.1007/ s00497-018-00357-2

Hsieh, T.-F., Ibarra, C. A., Silva, P., Zemach, A., Eshed-Williams, L., Fischer R. L. and Zilberman, D. (2009). Genome-wide demethylation of Arabidopsis endosperm. Science 324, 1451-1454. doi:10.1126/science.1172417

Hwang, B., Lee, J. H. and Bang, D. (2018). Single-cell RNA sequencing technologies and bioinformatics pipelines. Exp. Mol. Med. 50, 1-14. doi:10. 1038/s12276-018-0071-8

Ibarra, C. A., Feng, X., Schoft, V. K., Hsieh, T.-F., Uzawa, R., Rodrigues, J. A Zemach, A., Chumak, N., Machlicova, A., Nishimura, T. et al. (2012). Active DNA demethylation in plant companion cells reinforces transposon methylation in gametes. Science 337, 1360-1364. doi:10.1126/science.1224839

Ingouff, M., Rademacher, S., Holec, S., Šoljić, L., Xin, N., Readshaw, A., Foo, S. H., Lahouze, B., Sprunck, S. and Berger, F. (2010). Zygotic resetting of the HISTONE 3 variant repertoire participates in epigenetic reprogramming in Arabidopsis. Curr. Biol. 20, 2137-2143. doi:10.1016/j.cub.2010.11.012

Iwase, A., Harashima, H., Ikeuchi, M., Rymen, B., Ohnuma, M., Komaki, S. Morohashi, K., Kurata, T., Nakata, M., Ohme-Takagi, M. et al. (2017). WIND1 promotes shoot regeneration through transcriptional activation of ENHANCER OF SHOOT REGENERATION1 in Arabidopsis. Plant Cell 29, 54-69. doi:10.1105/tpc 16.00623

Jean-Baptiste, K., McFaline-Figueroa, J. L., Alexandre, C. M., Dorrity, M. W., Saunders, L., Bubb, K. L., Trapnell, C., Fields, S., Queitsch, C. and Cuperus, J. T. (2019). Dynamics of gene expression in single root cells of Arabidopsis thaliana. Plant Cell 31, 993-1011. doi:10.1105/tpc.18.00785

Jeong, S., Palmer, T. M. and Lukowitz, W. (2011). The RWP-RK facto GROUNDED promotes embryonic polarity by facilitating YODA MAP kinase signaling. Curr. Biol. 21, 1268-1276. doi:10.1016/j.cub.2011.06.049

Jullien, P. E., Susaki, D., Yelagandula, R., Higashiyama, T. and Berger, F. (2012). DNA methylation dynamics during sexual reproduction in Arabidopsis thaliana. Curr. Biol. 22, 1825-1830. doi:10.1016/j.cub.2012.07.061

Jullien, P. E., Bonnet, D. M. V., Pumplin, N., Schröeder, J. A. and Voinnet, O. (2020). Asymmetric expression of argonautes in Arabidopsis reproductive tissues. bioRxiv. doi:10.1101/2020.05.18.102863

Kao, P. and Nodine, M. D. (2019). Transcriptional activation of arabidopsis zygotes is required for initial cell divisions. Sci. Rep. 9, 17159. doi:10.1038/s41598-019 53704-2

Kirch, T., Simon, R., Grünewald, M. and Werr, W. (2003). The DORNRÖSCHEN/ ENHANCER OF SHOOT REGENERATION1 gene of arabidopsis acts in the control of meristem cell fate and lateral organ development. Plant Cell 15, 694-705. doi:10.1105/tpc.009480

Koi, S., Hisanaga, T., Sato, K., Shimamura, M., Yamato, K. T., Ishizaki, K. Kohchi, T. and Nakajima, K. (2016). An evolutionarily conserved plant RKD factor controls germ cell differentiation. Curr. Biol. 26, 1775-1781. doi:10.1016/j. cub.2016.05.013

Kőszegi, D., Johnston, A. J., Rutten, T., Czihal, A., Altschmied, L., Kumlehn, J. Wüst, S. E. J., Kirioukhova, O., Gheyselinck, J., Grossniklaus, U. et al. (2011) Members of the RKD transcription factor family induce an egg cell-like gene expression program: RKD controls the egg cell transcriptional program. Plant $\mathrm{J}$. 67, 280-291. doi:10.1111/j.1365-313X.2011.04592.x

Law, J. A. and Jacobsen, S. E. (2010). Establishing, maintaining and modifying DNA methylation patterns in plants and animals. Nat. Rev. Genet. 11, 204-220. doi:10.1038/nrg2719

Lee, M. T., Bonneau, A. R. and Giraldez, A. J. (2014). Zygotic genome activation during the maternal-to-zygotic transition. Annu. Rev. Cell Dev. Biol. 30, 581-613. doi:10.1146/annurev-cellbio-100913-013027

Lin, W., Sun, L., Huang, R.-Z., Liang, W., Liu, X., He, H., Fukuda, H., He, X.-Q. and Qian, W. (2020). Active DNA demethylation regulates tracheary element differentiation in Arabidopsis. Sci. Adv. 6, eaaz2963 doi:10.1126/sciadv aaz2963

Long, Y., Liu, Z., Jia, J., Mo, W., Fang, L., Lu, D., Liu, B., Zhang, H., Chen, W. and Zhai, J. (2021). FlsnRNA-seq: protoplasting-free full-length single-nucleus RNA profiling in plants. Genome Biol. 22, 66. doi:10.1186/s13059-021-02288-0 
Lotan, T., Ohto, M.-a., Yee, K. M., West, M. A. L., Lo, R., Kwong, R. W. Yamagishi, K., Fischer, R. L., Goldberg, R. B. and Harada, J. J. (1998). Arabidopsis LEAFY COTYLEDON1 is sufficient to induce embryo development in vegetative cells. Cell 93, 1195-1205. doi:10.1016/S0092-8674(00)81463-4

Lukowitz, W., Roeder, A., Parmenter, D. and Somerville, C. (2004). A MAPKK Kinase gene regulates extra-embryonic cell fate in Arabidopsis. Cell 116 109-119. doi:10.1016/S0092-8674(03)01067-5

Mansfield, S. G. and Briarty, L. G. (1991). Early embryogenesis in Arabidopsis thaliana. II. The developing embryo. Can. J. Bot. 69, 461-476. doi:10.1139/b91063

Martin, M. (2011). Cutadapt removes adapter sequences from high-throughput sequencing reads. EMBnet. J. 17, 10-12. doi:10.14806/ej.17.1.200

Mayer, K. F. X., Schoof, H., Haecker, A., Lenhard, M., Jürgens, G. and Laux, T. (1998). Role of WUSCHEL in regulating stem cell fate in the Arabidopsis shoo meristem. Cell 95, 805-815. doi:10.1016/S0092-8674(00)81703-1

Mclnnes, L., Healy, J. and Melville, J. (2018). UMAP: uniform manifold approximation and projection for dimension reduction. arXiv [stat.ML].

McLeay, R. C. and Bailey, T. L. (2010). Motif enrichment analysis: a unified framework and an evaluation on ChIP data. BMC Bioinform. 11, 165. doi:10.1186/ 1471-2105-11-165

Meinke, D. W. (2020). Genome-wide identification of EMBRYO-DEFECTIVE (EMB) genes required for growth and development in Arabidopsis. New Phytol. 226 306-325.

Menges, M., Hennig, L., Gruissem, W. and Murray, J. A. H. (2003). Genome-wide gene expression in an Arabidopsis cell suspension. Plant Mol. Biol. 53, 423-442. doi:10.1023/B:PLAN.0000019059.56489.ca

Meyerowitz, E. M. (2002). Plants compared to animals: the broadest comparative study of development. Science 295, 1482-1485. doi:10.1126/science.1066609

Mi, H., Ebert, D., Muruganujan, A., Mills, C., Albou, L.-P., Mushayamaha, T. and Thomas, P. D. (2021). PANTHER version 16: a revised family classification, treebased classification tool, enhancer regions and extensive API. Nucleic Acids Res. 49, D394-D403. doi:10.1093/nar/gkaa1106

Mosher, R. A. and Melnyk, C. W. (2010). siRNAs and DNA methylation: seedy epigenetics. Trends Plant Sci. 15, 204-210. doi:10.1016/j.tplants.2010.01.002

Muralla, R., Lloyd, J. and Meinke, D. (2011). Molecular foundations of reproductive lethality in Arabidopsis thaliana. PLoS ONE 6, e28398. doi:10.1371/journal.pone. 0028398

Nagasaki, H., Itoh, J.-I., Hayashi, K., Hibara, K.-I., Satoh-Nagasawa, N., Nosaka M., Mukouhata, M., Ashikari, M., Kitano, H., Matsuoka, M. et al. (2007). The small interfering RNA production pathway is required for shoot meristem initiation in rice. Proc. Natl. Acad. Sci. USA 104, 14867-14871. doi:10.1073/pnas. 0704339104

Nagl, W. (1990). Translocation of Putrescine in the ovule, suspensor and embryo of Phaseolus coccineus. J. Plant Physiol. 136, 587-591. doi:10.1016/S0176 1617(11)80218-X

Nodine, M. D. and Bartel, D. P. (2012). Maternal and paternal genomes contribute equally to the transcriptome of early plant embryos. Nature 482, 94-97. doi:10. 1038/nature10756

Nodine, M. D., Yadegari, R. and Tax, F. E. (2007). RPK1 and TOAD2 are two receptor-like kinases redundantly required for arabidopsis embryonic pattern formation. Dev. Cell 12, 943-956. doi:10.1016/j.devcel.2007.04.003

Ogawa, E., Yamada, Y., Sezaki, N., Kosaka, S., Kondo, H., Kamata, N., Abe, M. Komeda, Y. and Takahashi, T. (2015). ATML1 and PDF2 play a redundant and essential role in Arabidopsis embryo development. Plant Cell Physiol. 56 1183-1192. doi:10.1093/pcp/pcv045

Palovaara, J., de Zeeuw, T. and Weijers, D. (2016). Tissue and organ initiation in the plant embryo: a first time for everything. Annu. Rev. Cell Dev. Biol. 32, 47-75 doi:10.1146/annurev-cellbio-111315-124929

Palovaara, J., Saiga, S., Wendrich, J. R., van 't Wout Hofland, N., van Schayck, J. P., Hater, F., Mutte, S., Sjollema, J., Boekschoten, M., Hooiveld, G. J. et al. (2017). Transcriptome dynamics revealed by a gene expression atlas of the early Arabidopsis embryo. Nat. Plants 3, 894-904. doi:10.1038/s41477-017-0035-3

Papareddy, R. K., Páldi, K., Paulraj, S., Kao, P., Lutzmayer, S. and Nodine, M. D. (2020). Chromatin regulates expression of small RNAs to help maintain transposon methylome homeostasis in Arabidopsis. Genome Biol. 21, 251 doi:10.1186/s13059-020-02163-4

Penterman, J., Zilberman, D., Huh, J. H., Ballinger, T., Henikoff, S. and Fischer, R. L. (2007). DNA demethylation in the Arabidopsis genome. Proc. Natl. Acad Sci. USA 104, 6752-6757. doi:10.1073/pnas.0701861104

Picard, C. L., Povilus, R. A., Williams, B. P. and Gehring, M. (2021) Transcriptional and imprinting complexity in Arabidopsis seeds at singlenucleus resolution. Nat. Plants 7, 730-738. doi: 10.1038/s41477-021-00922-0

Picelli, S., Faridani, O. R., Björklund, Å. K., Winberg, G., Sagasser, S. and Sandberg, R. (2014a). Full-length RNA-seq from single cells using Smart-seq2. Nat. Protoc. 9, 171-181. doi:10.1038/nprot.2014.006

Picelli, S., Björklund, Å. K., Reinius, B., Sagasser, S., Winberg, G. and Sandberg, R. (2014b). Tn5 transposase and tagmentation procedures for massively scaled sequencing projects. Genome Res. 24, 2033-2040. doi:10. $1101 /$ gr. 177881.114
Pikaard, C. S. and Mittelsten Scheid, O. (2014). Epigenetic regulation in plants Cold Spring Harb. Perspect. Biol. 6, a019315. doi:10.1101/cshperspect.a019315

Plotnikova, A., Kellner, M. J., Schon, M. A., Mosiolek, M. and Nodine, M. D. (2019). MicroRNA dynamics and functions during Arabidopsis embryogenesis. Plant Cell 31, 2929-2946. doi:10.1105/tpc.19.00395

Qiu, X., Hill, A., Packer, J., Lin, D., Ma, Y.-A. and Trapnell, C. (2017). Single-cell mRNA quantification and differential analysis with census. Nat. Methods 14 309-315. doi:10.1038/nmeth.4150

Riechmann, J. L. (2002). Transcriptional regulation: a genomic overview. Arabidopsis Book 2002, e0085. doi:10.1199/tab.0085

Robert, H. S., Park, C., Gutièrrez, C. L., Wójcikowska, B., Pěnčík, A., Novák, O. Chen, J., Grunewald, W., Dresselhaus, T., Friml, J. et al. (2018). Maternal auxin supply contributes to early embryo patterning in Arabidopsis. Nat. Plants 4 548-553. doi:10.1038/s41477-018-0204-z

Ryu, K. H., Huang, L., Kang, H. M. and Schiefelbein, J. (2019). Single-cell RNA sequencing resolves molecular relationships among individual plant cells. Plant Physiol. 179, 1444-1456. doi:10.1104/pp.18.01482

Satterlee, J. W., Strable, J. and Scanlon, M. J. (2020). Plant stem-cell organization and differentiation at single-cell resolution. Proc. Natl. Acad. Sci. USA 117 33689-33699. doi:10.1073/pnas.2018788117

Schon, M. A. and Nodine, M. D. (2017). Widespread contamination of Arabidopsis embryo and endosperm transcriptome data sets. Plant Cell 29, 608-617. doi:10. 1105/tpc.16.00845

Shi, C., Luo, P., Du, Y.-T., Chen, H., Huang, X., Cheng, T.-H., Luo, A., Li, H.-J. Yang, W.-C., Zhao, P. et al. (2019). Maternal control of suspensor programmed cell death via gibberellin signaling. Nat. Commun. 10, 3484. doi:10.1038/s41467019-11476-3

Shulse, C. N., Cole, B. J., Ciobanu, D., Lin, J., Yoshinaga, Y., Gouran, M., Turco, G. M., Zhu, Y., O'Malley, R. C., Brady, S. M. et al. (2019). High-throughput singlecell transcriptome profiling of plant cell types. Cell Rep. 27, 2241-2247.e4. doi:10. 1016/j.celrep.2019.04.054

Slane, D., Kong, J., Berendzen, K. W., Kilian, J., Henschen, A., Kolb, M. Schmid, M., Harter, K., Mayer, U., De Smet, I. et al. (2014). Cell type-specific transcriptome analysis in the early Arabidopsis thaliana embryo. Development 141, 4831-4840. doi:10.1242/dev.116459

Slotkin, R. K., Vaughn, M., Borges, F., Tanurdžić, M., Becker, J. D., Feijó, J. A. and Martienssen, R. A. (2009). Epigenetic reprogramming and small RNA silencing of transposable elements in pollen. Cell 136, 461-472. doi:10.1016/j.cell. 2008.12.038

Smit, M. E., Llavata-Peris, C. I., Roosjen, M., van Beijnum, H., Novikova, D. Levitsky, V., Sevilem, I., Roszak, P., Slane, D., Jürgens, G. et al. (2020) Specification and regulation of vascular tissue identity in the Arabidopsis embryo. Development 147, dev186130. doi:10.1242/dev.186130

Smyth, D. R., Bowman, J. L. and Meyerowitz, E. M. (1990). Early flower development in Arabidopsis. Plant Cell 2, 755-767. doi:10.1105/tpc.2.8.755

Song, Q., Ando, A., Jiang, N., Ikeda, Y. and Chen, Z. J. (2020). Single-cell RNAseq analysis reveals ploidy-dependent and cell-specific transcriptome changes in Arabidopsis female gametophytes. Genome Biol. 21, 178. doi:10.1186/s13059 020-02094-0

Stadler, R., Lauterbach, C. and Sauer, N. (2005). Cell-to-cell movement of green fluorescent protein reveals post-phloem transport in the outer integument and identifies symplastic domains in Arabidopsis seeds and embryos. Plant Physiol. 139, 701-712. doi:10.1104/pp.105.065607

Tadros, W. and Lipshitz, H. D. (2009). The maternal-to-zygotic transition: a play in two acts. Development 136, 3033-3042. doi:10.1242/dev.033183

Tian, F., Yang, D.-C., Meng, Y.-Q., Jin, J. and Gao, G. (2020). PlantRegMap: charting functional regulatory maps in plants. Nucleic Acids Res. 48 D1104-D1113. doi:10.1093/nar/gkz1020

Trapnell, C., Cacchiarelli, D., Grimsby, J., Pokharel, P., Li, S., Morse, M., Lennon, N. J., Livak, K. J., Mikkelsen, T. S. and Rinn, J. L. (2014). The dynamics and regulators of cell fate decisions are revealed by pseudotemporal ordering of single cells. Nat. Biotechnol. 32, 381-386. doi:10.1038/nbt.2859

Tucker, M. R., Hinze, A., Tucker, E. J., Takada, S., Jürgens, G. and Laux, T. (2008). Vascular signalling mediated by ZWILLE potentiates WUSCHEL function during shoot meristem stem cell development in the Arabidopsisembryo. Development 135, 2839-2843. doi:10.1242/dev.023648

Ueda, M., Zhang, Z. and Laux, T. (2011). Transcriptional activation of Arabidopsis axis patterning genes WOX8/9 links zygote polarity to embryo development. Dev. Cell 20, 264-270. doi:10.1016/j.devcel.2011.01.009

Ueda, M., Aichinger, E., Gong, W., Groot, E., Verstraeten, I., Vu, L. D., De Smet I., Higashiyama, T., Umeda, M. and Laux, T. (2017). Transcriptional integration of paternal and maternal factors in the Arabidopsis zygote. Genes Dev. 31 617-627. doi:10.1101/gad.292409.116

Vatén, A., Dettmer, J., Wu, S., Stierhof, Y.-D., Miyashima, S., Yadav, S. R. Roberts, C. J., Campilho, A., Bulone, V., Lichtenberger, R. et al. (2011) Callose biosynthesis regulates symplastic trafficking during root development. Dev. Cell 21, 1144-1155. doi:10.1016/j.devcel.2011.10.006

Waki, T., Hiki, T., Watanabe, R., Hashimoto, T. and Nakajima, K. (2011). The Arabidopsis RWP-RK protein RKD4 triggers gene expression and pattern 
formation in early embryogenesis. Curr. Biol. 21, 1277-1281. doi:10.1016/j.cub. 2011.07.001

Weirauch, M. T., Yang, A., Albu, M., Cote, A. G., Montenegro-Montero, A. Drewe, P., Najafabadi, H. S., Lambert, S. A., Mann, I., Cook, K. et al. (2014). Determination and inference of eukaryotic transcription factor sequence specificity. Cell 158, 1431-1443. doi:10.1016/j.cell.2014.08.009

Welch, D., Hassan, H., Blilou, I., Immink, R., Heidstra, R. and Scheres, B. (2007). Arabidopsis JACKDAW and MAGPIE zinc finger proteins delimit asymmetric cell division and stabilize tissue boundaries by restricting SHORT-ROOT action. Genes Dev. 21, 2196-2204. doi:10.1101/gad.440307

Xiang, D., Venglat, P., Tibiche, C., Yang, H., Risseeuw, E., Cao, Y., Babic, V., Cloutier, M., Keller, W., Wang, E. et al. (2011). Genome-wide analysis reveals gene expression and metabolic network dynamics during embryo development in Arabidopsis. Plant Physiol. 156, 346-356. doi:10.1104/pp.110.171702

Xiao, J., Jin, R., Yu, X., Shen, M., Wagner, J. D., Pai, A., Song, C., Zhuang, M., Klasfeld, S., He, C. et al. (2017). Cis and trans determinants of epigenetic silencing by Polycomb repressive complex 2 in Arabidopsis. Nat. Genet. 49, 1546-1552. doi:10.1038/ng.3937

Xu, X., Crow, M., Rice, B. R., Li, F., Harris, B., Liu, L., Demesa-Arevalo, E., Lu, Z., Wang, L., Fox, N. et al. (2021). Single-cell RNA sequencing of developing maize ears facilitates functional analysis and trait candidate gene discovery. Dev. Cell. 56, 557-568.e6. doi:10.1016/j.devcel.2020.12.015

Yeung, E. C. (1980). Embryogeny of Phaseolus: the role of the suspensor. Z. Pflanzenphysiol. 96, 17-28. doi:10.1016/S0044-328X(80)80096-1
Zhang, T.-Q., Xu, Z.-G., Shang, G.-D. and Wang, J.-W. (2019). A single-cell RNA sequencing profiles the developmental landscape of arabidopsis root. Mol. Plant 12, 648-660. doi:10.1016/j.molp.2019.04.004

Zhao, J., Xin, H., Qu, L., Ning, J., Peng, X., Yan, T., Ma, L., Li, S. and Sun, M.-X. (2011). Dynamic changes of transcript profiles after fertilization are associated with de novo transcription and maternal elimination in tobacco zygote, and mark the onset of the maternal-to-zygotic transition. Plant J. 65, 131-145. doi:10.1111/ j.1365-313X.2010.04403.x

Zhao, P., Zhou, X., Shen, K., Liu, Z., Cheng, T., Liu, D., Cheng, Y., Peng, X. and Sun, M.-X. (2019). Two-step maternal-to-Zygotic transition with two-phase parental genome contributions. Dev. Cell 49, 882-893.e5. doi:10.1016/j.devcel. 2019.04.016

Zhou, Y., Hartwig, B., James, G. V., Schneeberger, K. and Turck, F. (2016). Complementary Activities of TELOMERE REPEAT BINDING proteins and polycomb group complexes in transcriptional regulation of target genes. Plant Cell 28, 87-101. doi:10.1105/tpc.15.00787

Zhou, Y., Wang, Y., Krause, K., Yang, T., Dongus, J. A., Zhang, Y. and Turck, F. (2018). Telobox motifs recruit CLF/SWN-PRC2 for H3K27me3 deposition via TRB factors in Arabidopsis. Nat. Genet. 50, 638-644. doi:10.1038/s41588-0180109-9

Zhou, X., Liu, Z., Shen, K., Zhao, P. and Sun, M.-X. (2020). Cell lineage-specific transcriptome analysis for interpreting cell fate specification of proembryos. Nat. Commun. 11, 1366. doi:10.1038/s41467-020-15189-w 
A

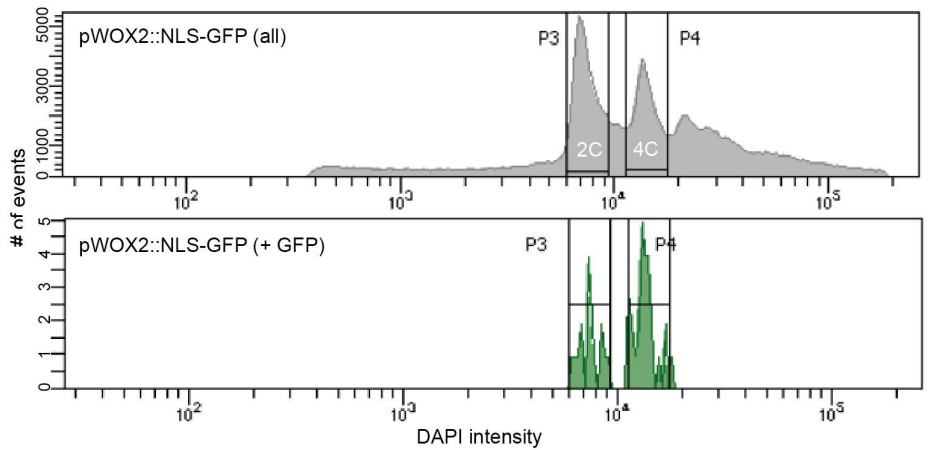

B

C

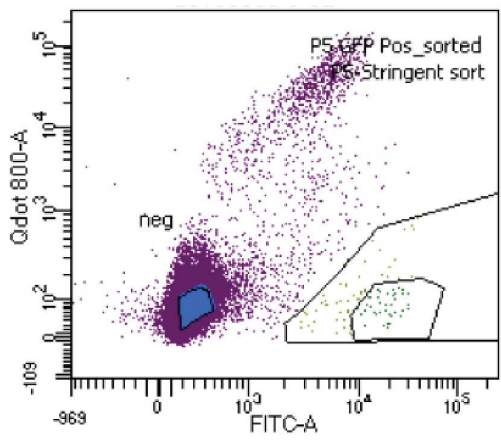

D
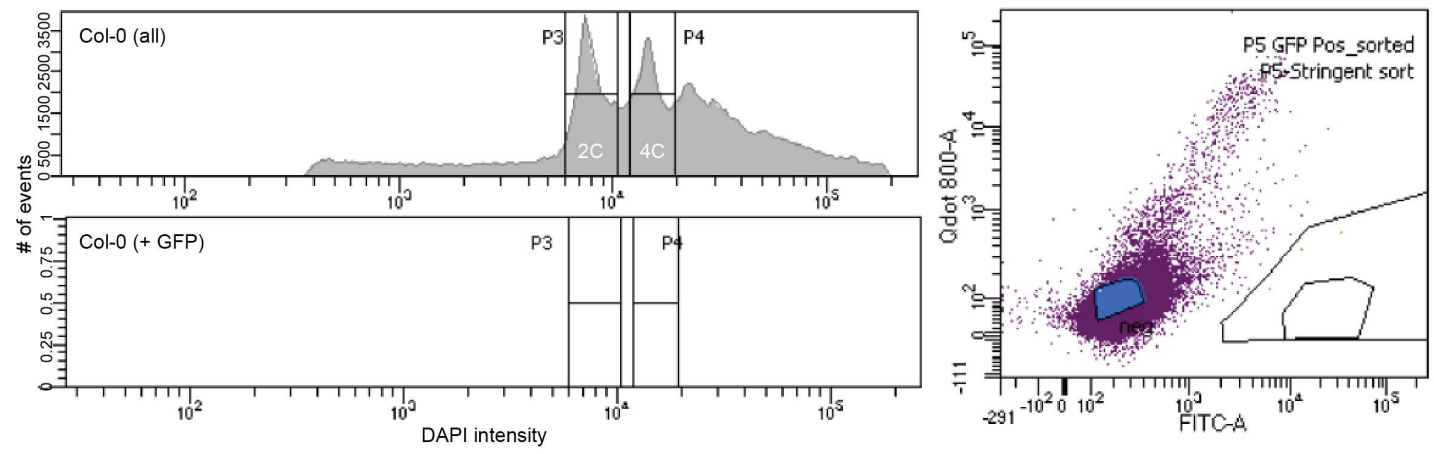

E
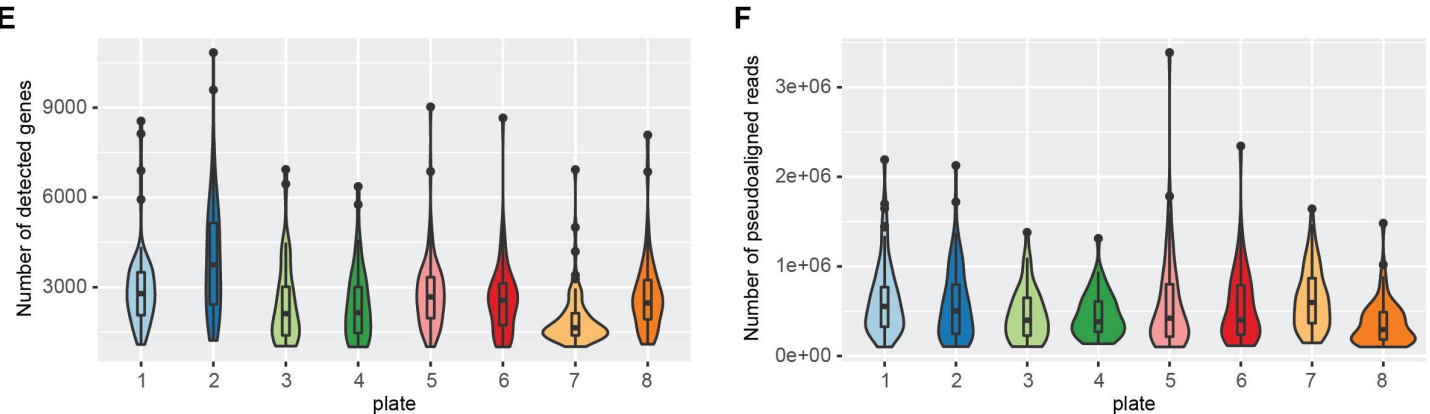

Figure S1. Embryonic nuclei enrichment with FACS and qualities of snRNA-seq libraries. (A and C) Representative DAPI (top) and GFP (bottom) profiles for the pWOX2::NLS-GFP transgenic line $(A)$ and Col-0 (C). DAPI intensities and the number of events, which include nuclei, are shown on the $x$ and $y$ axes, respectively. Selection gates centered on the $2 \mathrm{C}$ and $4 \mathrm{C}$ peaks were applied to reduce debris or aggregates. (B and D) Representative fluorescence scatter plots for the pWOX2::NLS-GFP transgenic line (B) and Col-0 (D), where the $x$ and $y$ axes represent GFP emission and 800-nm auto-fluorescence, respectively. Each dot represents an event (e.g. a nucleus) that passed the DAPI 2C/4C gate. Two regions representing high GFP and low infrared fluorescence were drawn, and the more stringent inner circle was used to enrich GFP-positive nuclei. ( $E$ and F) The number of genes detected (E) and reads aligned to the reference transcriptome $(F)$ in single nuclear libraries from each 96-well plate. 


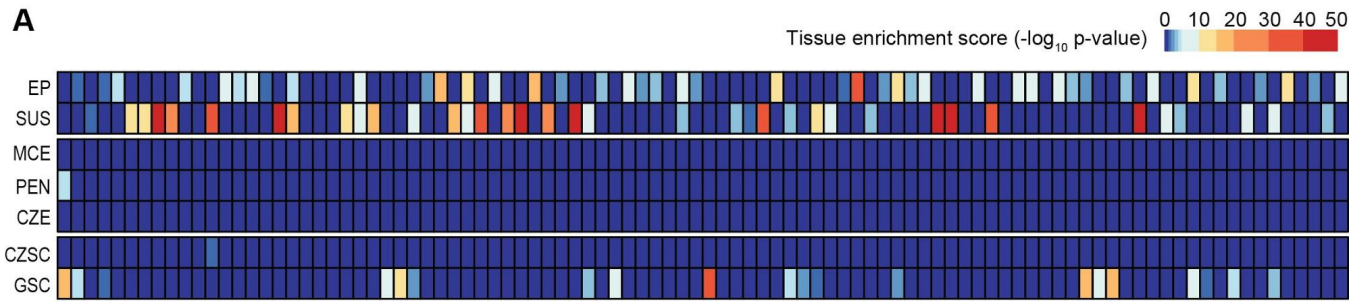

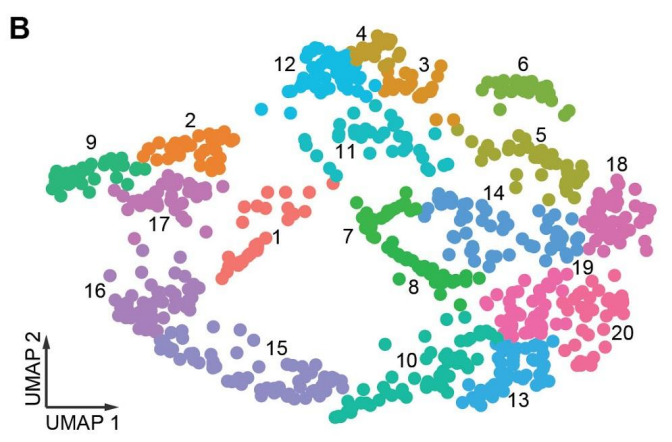

D
C

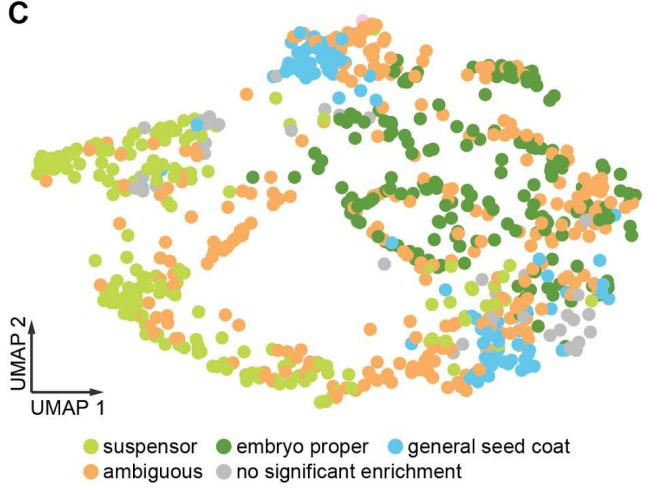

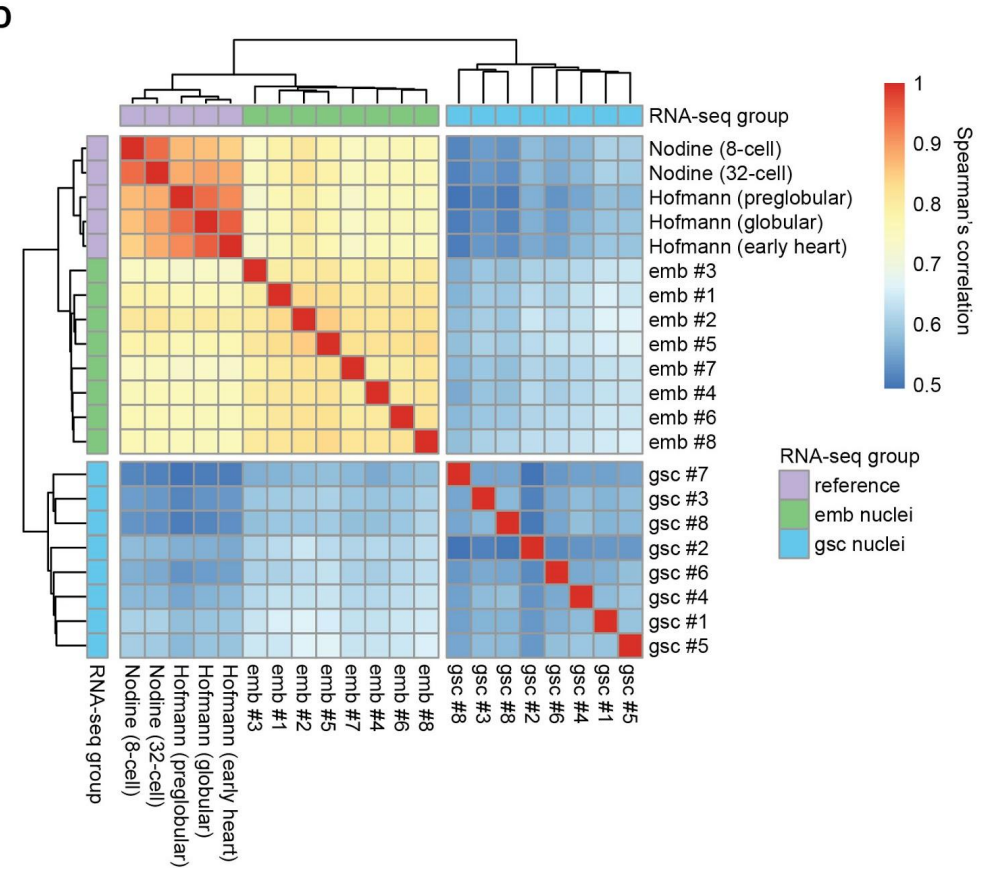

Figure S2. Assessing and mitigating maternal RNA contamination. (A) A representative result of tissue enrichment tests on snRNA-seq libraries from a 96-well plate. Each row represents one of the seed tissue types, and each column represents a snRNA-seq transcriptome. Most nuclei were enriched for one tissue type, indicating that the major source of maternal contamination were false-positive sorted maternal nuclei instead of the ambient RNA. EP, embryo proper; SUS, suspensor; MCE, micropylar endosperm; PEN, peripheral endosperm; CZE, chalazal endosperm; CZSC, chalazal seed coat; GSC, general seed coat. 
$(B, C)$ Unsupervised clustering and identification of contaminated nuclei. All snRNA-seq libraries with $\geq 100,000$ aligned reads and $\geq 1,000$ expressed genes were clustered and the resulting UMAP plots were color-coded by clusters $(B)$ or the significantly enriched tissue type according to tissue enrichment test (C). If a snRNA-seq library had no significantly enriched tissue type or was significantly enriched for more than one tissue type, it was labeled as no significant enrichment or ambiguous, respectively. (D) Heatmap illustrating Spearman's correlation coefficients among the snRNA-seq libraries classified as embryonic (emb) or general seed coat (gsc) and grouped by plate as shown Fig. 1C and published embryonic datasets (Hofmann et al., 2019; Nodine and Bartel, 2012). 
A
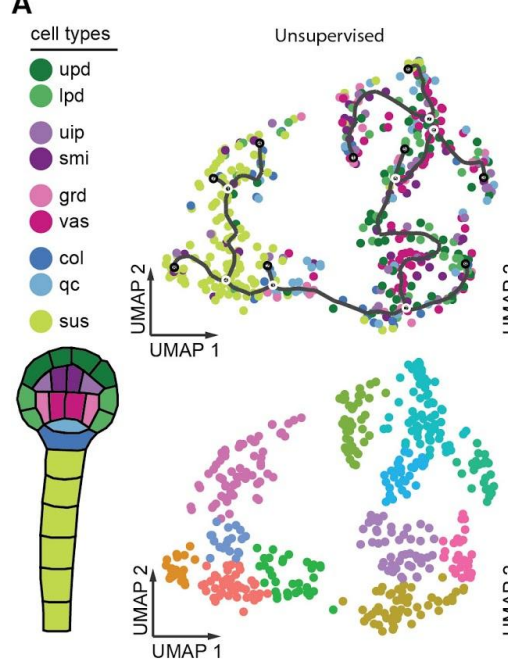

C

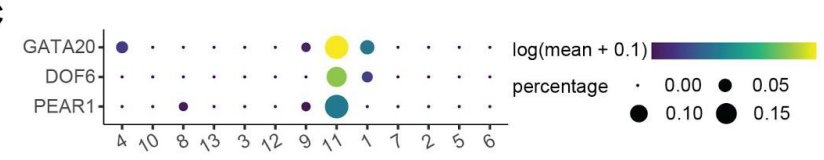

D

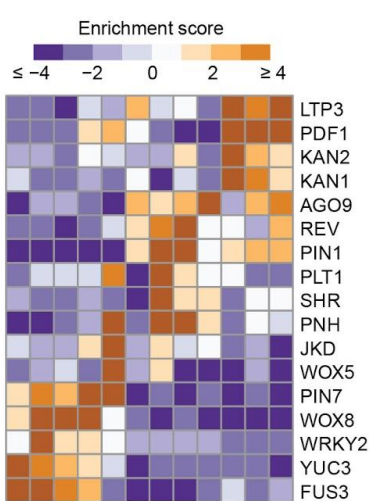

E
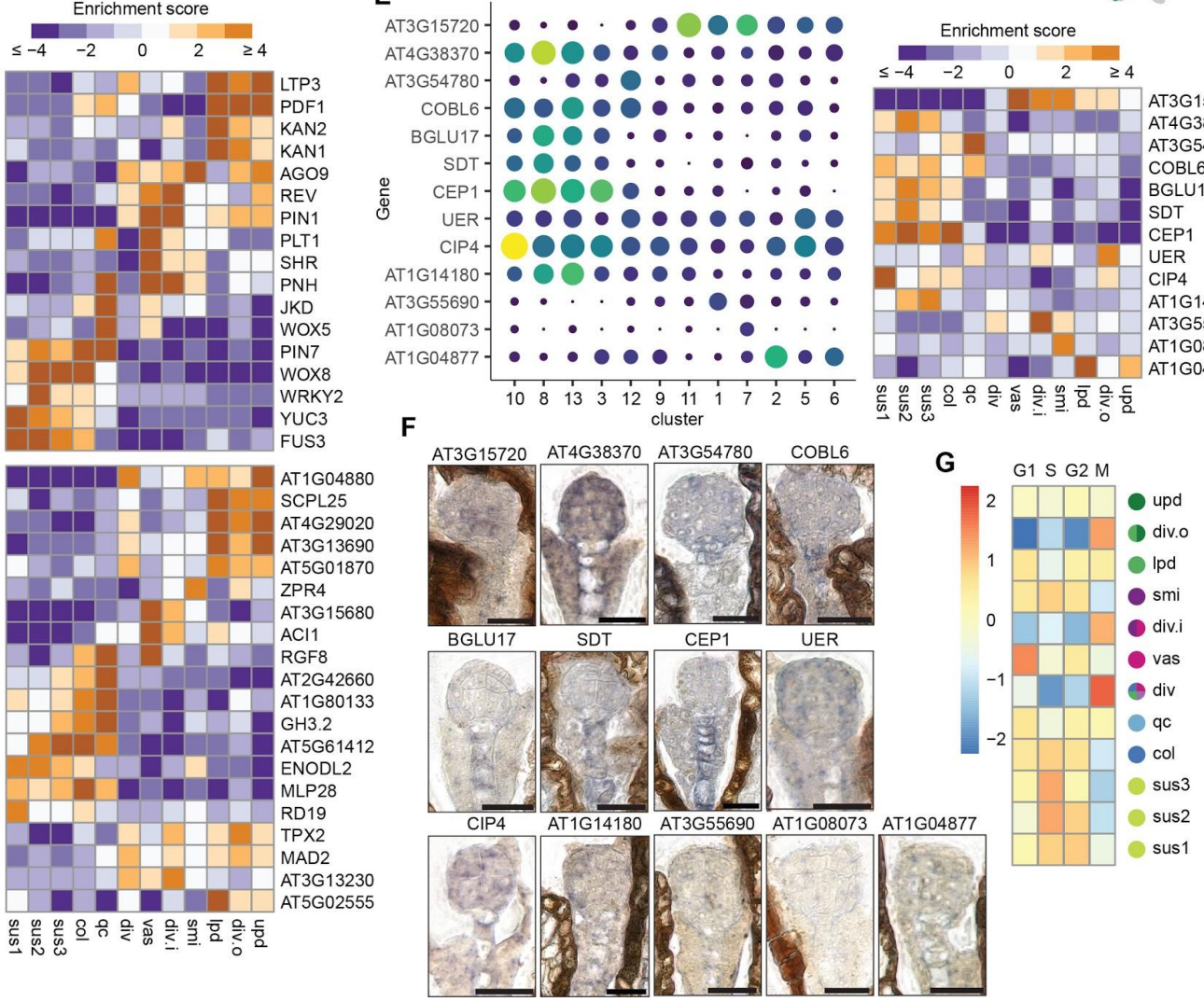

B
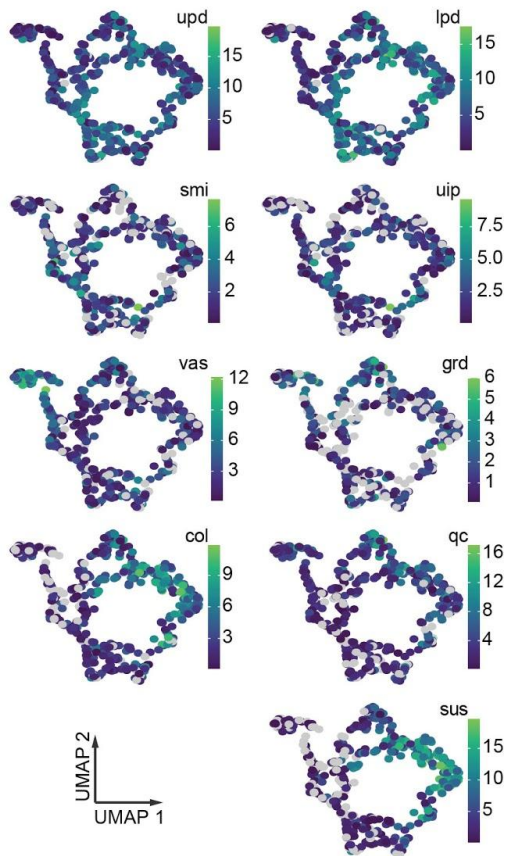

Figure S3. Resolving cell types by clustering. (A) UMAP plots based on unsupervised (left) and clustering based on expression of marker genes (right). The embryonic cell types are as in Fig. 2A. Each dot represents a nucleus and was colored according to cell type scores (top) or cluster (bottom). (B) Accumulated expression of cell-enriched markers across clusters defined in 
Fig. 2A and B. Each dot represents a nucleus and was colored according to expression levels (i.e. accumulated census counts) based on the keys. Cell types are indicated in each graph and abbreviations are as in Fig. 2A. (C) Dot plots illustrating the expression patterns across the clusters defined in Fig. 2A and B for three vascular-specific genes (Smit et al., 2020), which were not included in the marker list used to guide the clustering. The sizes of dots represent the percentage of nuclei the transcript was detected in for each cluster, and the colors represent the $\log _{10}$-transformed mean expression levels of each cluster. (D) Enrichment scores of the transcripts presented in Fig. 2C (top) and Fig. 2E (bottom). Cluster identities are indicated at the bottom and are as in Fig. 2G. (E) Dot plots of expression patterns (left) and heatmaps of enrichment scores (right) corresponding to the remaining 13 RNA ISH candidates not shown in Fig. 2. (F) Representative RNA ISH images of the remaining 13 candidates not shown in Fig. 2. Scale bars represent $20 \mu \mathrm{m}$. (G) Enrichments and depletions of cell-cycle related genes among the top-250 ranked genes for each cluster. Cluster identities are as in Fig. $1 \mathrm{G}$. 


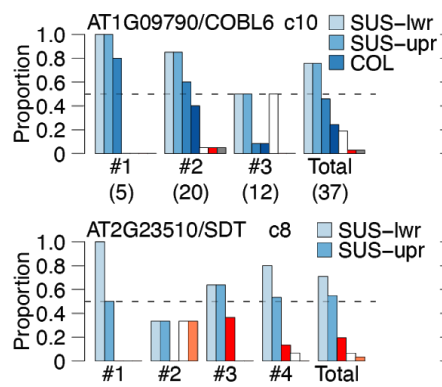

(2) (3) (11) (15) (31)
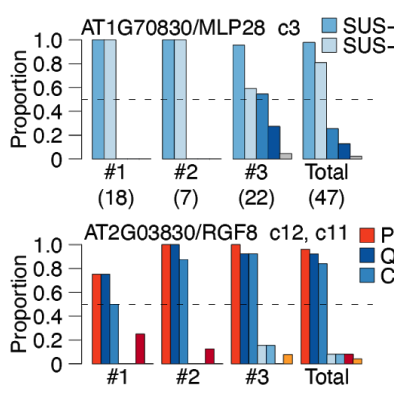

$\begin{array}{llll}(4) & \text { (8) (13) (25) }\end{array}$
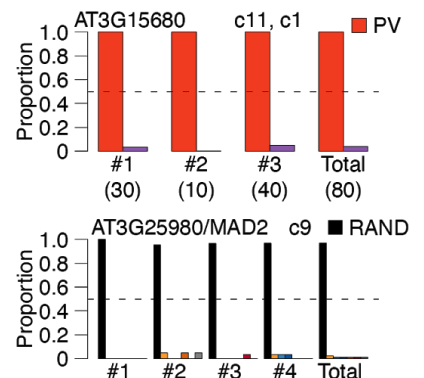

(14) (21) (30) (31) (96)

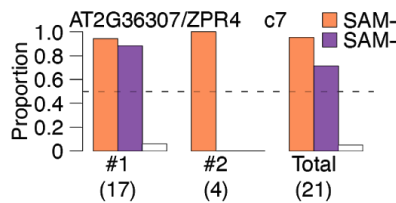

1.0 AT5G02555 C2 $\square$ ND

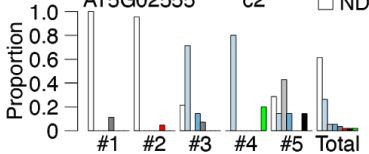

(9) (22) (14) (5) (7) (57)

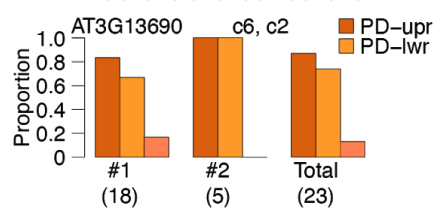

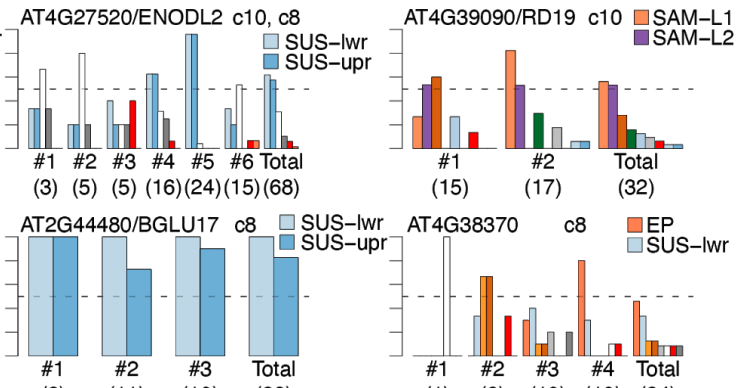

(1) (3) (10) (10) (24)

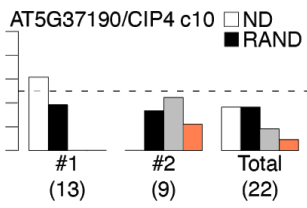

AT1G14180 c13

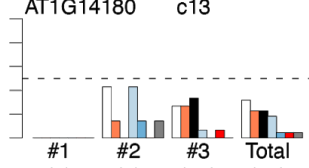

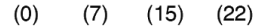
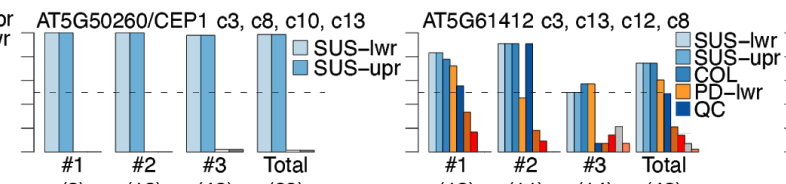
$\begin{array}{cccc}\# 1 & \# 2 & \# 3 & \text { Total } \\ (18) & (11) & (14) & (43)\end{array}$ AT1G80133 c12, c3 $\because \mathrm{COL}$

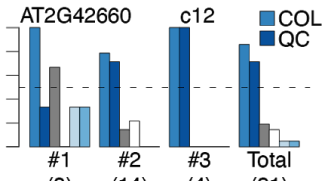

(3) (14) (4) (21)
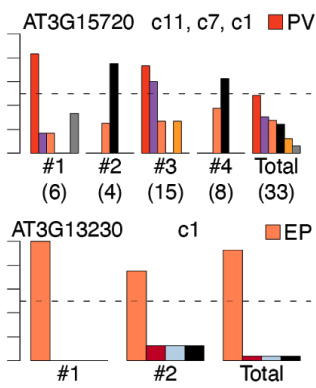

(20) (8) (28)
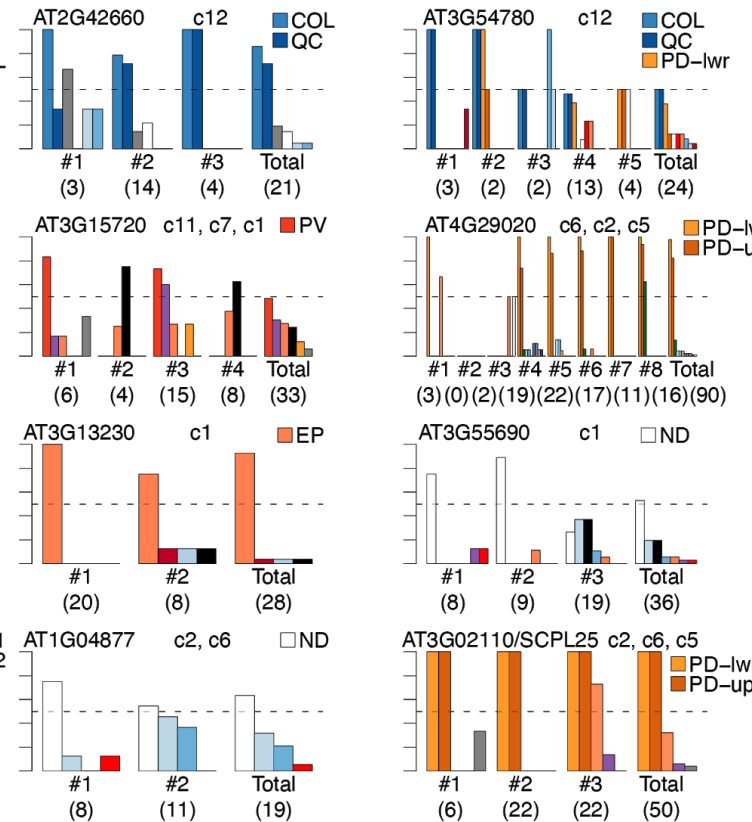

(3) (2) (2) (13) (4) (24)
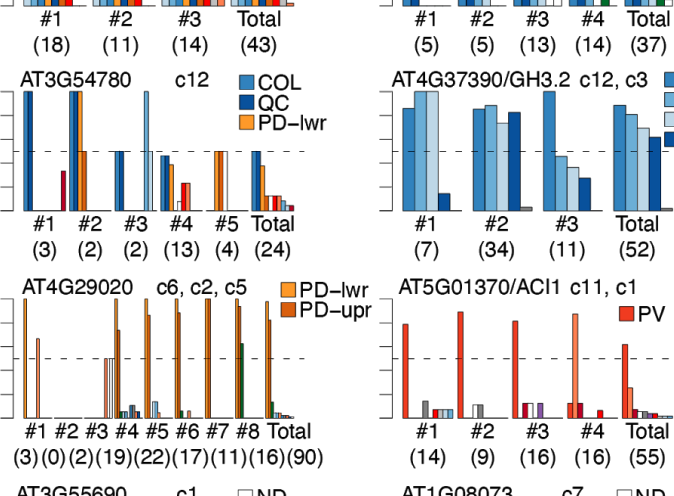

(5) (5) (13) (14) (37)

AT4G37390/GH3.2 c12, C3 $\square \mathrm{COL}$

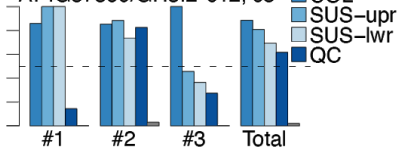

(7) (34) (11) (52)

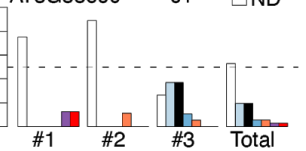

(8) (9) (19) (36)
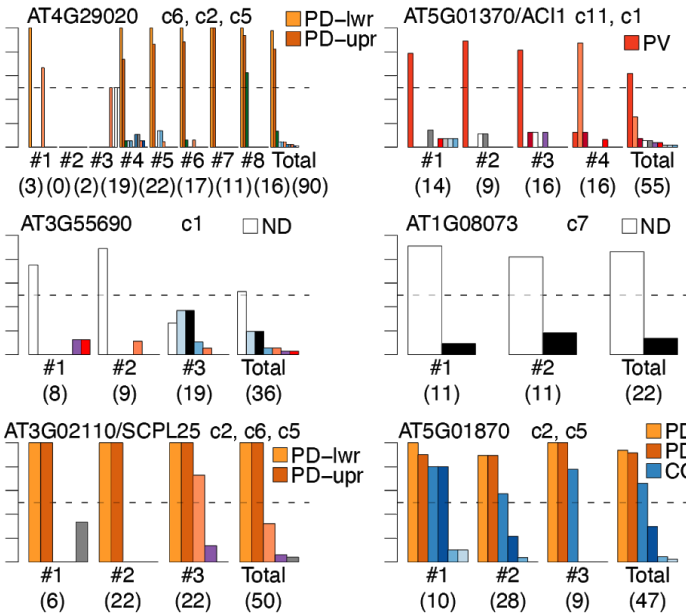

(11) (11) (22)
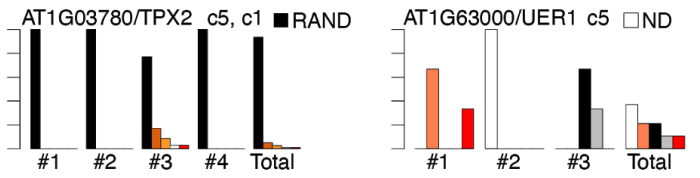

(47) (2) (35) (38) (122)
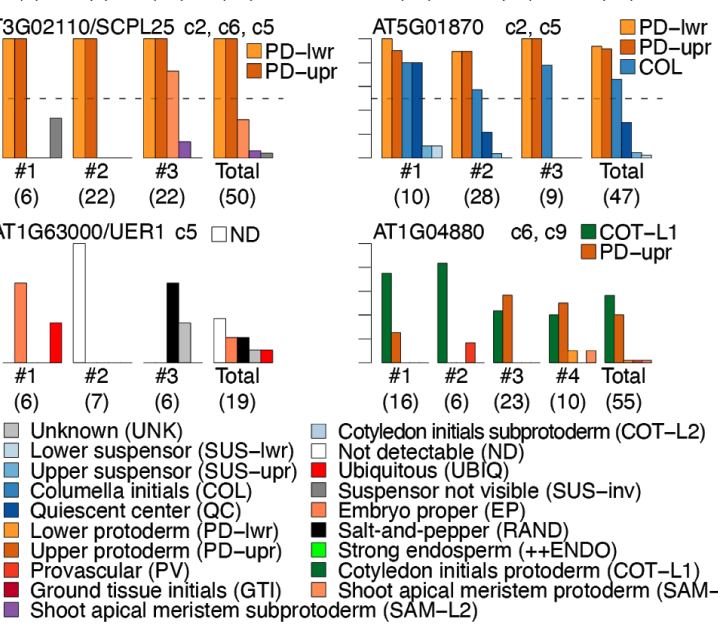

(16) (6) (23) (10) (55)

Cotyledon initials subprotoderm (COT-L2)
Not detectable (ND)

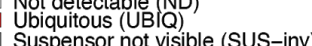

Suspensor not visible (SUS-inv)

Embryo proper (EP)

Strong endosperm (++ENDO)

Cotyledon initials protoderm (COT-L1)

Shoot apical meristem protoderm (SAM-L1) Shoot apical meristem subprotoderm (SAM-L2)

Figure S4. Quantification of RNA in situ patterns. Bar plots illustrating the proportion of globular-stage embryos with RNA in situ signals in various cell types. Each panel summarizes patterns observed for individual transcripts. Corresponding unique gene identifiers (i.e. Arabidopsis Genome Initiative identifiers; AGIs) and, if annotated, common names are shown in the upper-left corner of each panel. Clusters for which transcripts are within the top-250 ranked 
genes are indicated at the top of each graph (e.g. c1, c2, etc.) in ascending order based on their rankings. Proportions of signals are shown for individual slides (i.e. technical replicates), as well as totals for each transcript, and the number of embryos examined (i.e. biological replicates) are noted in parentheses. Legends in each panel are shown for patterns that occurred in $>33 \%$ of embryos, and the full legend is in the bottom-right corner. 
A

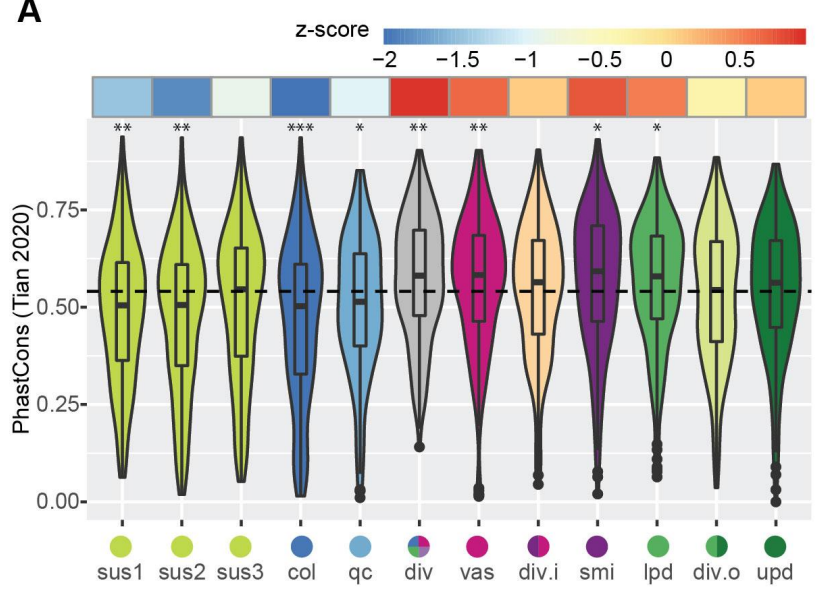

B

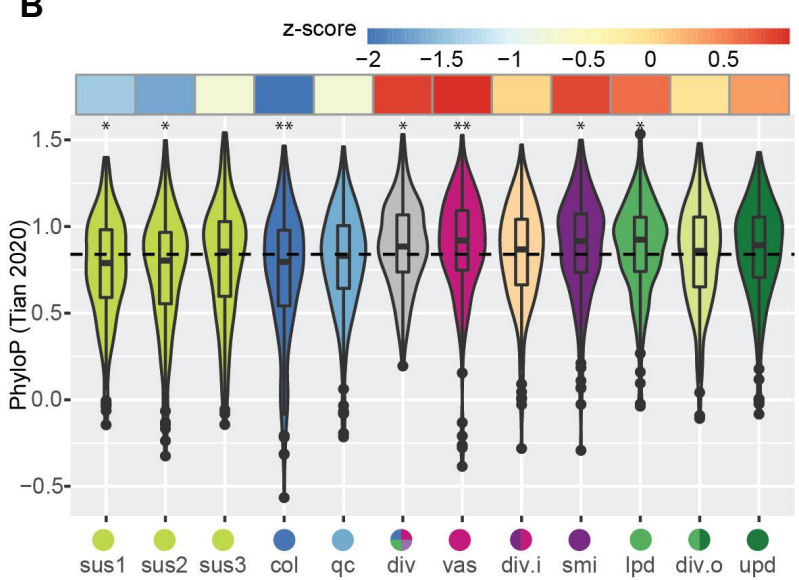

Figure S5. Conservation score distribution among clusters.

(A and B) PhastCons (A) and PhyloP (B) conservation scores (Tian et al., 2020) of the top-250 ranked genes for each cluster. The mean scores of all expressed genes are indicated by dashed lines, deviations from the means are presented in the upper row as z-scores. The asterisks indicate p-values $\leq 0.05\left(^{*}\right), \leq 0.01\left(^{* *}\right)$ or $\leq 0.001\left(^{* * *}\right)$ based on two-sided Kolmogorov-Smirnov tests with the alternative hypothesis that the cluster conservation score distributions of the top-ranked 250 genes were not equal to that of all expressed genes in embryos. Cluster identities are as in Fig. 2G. 


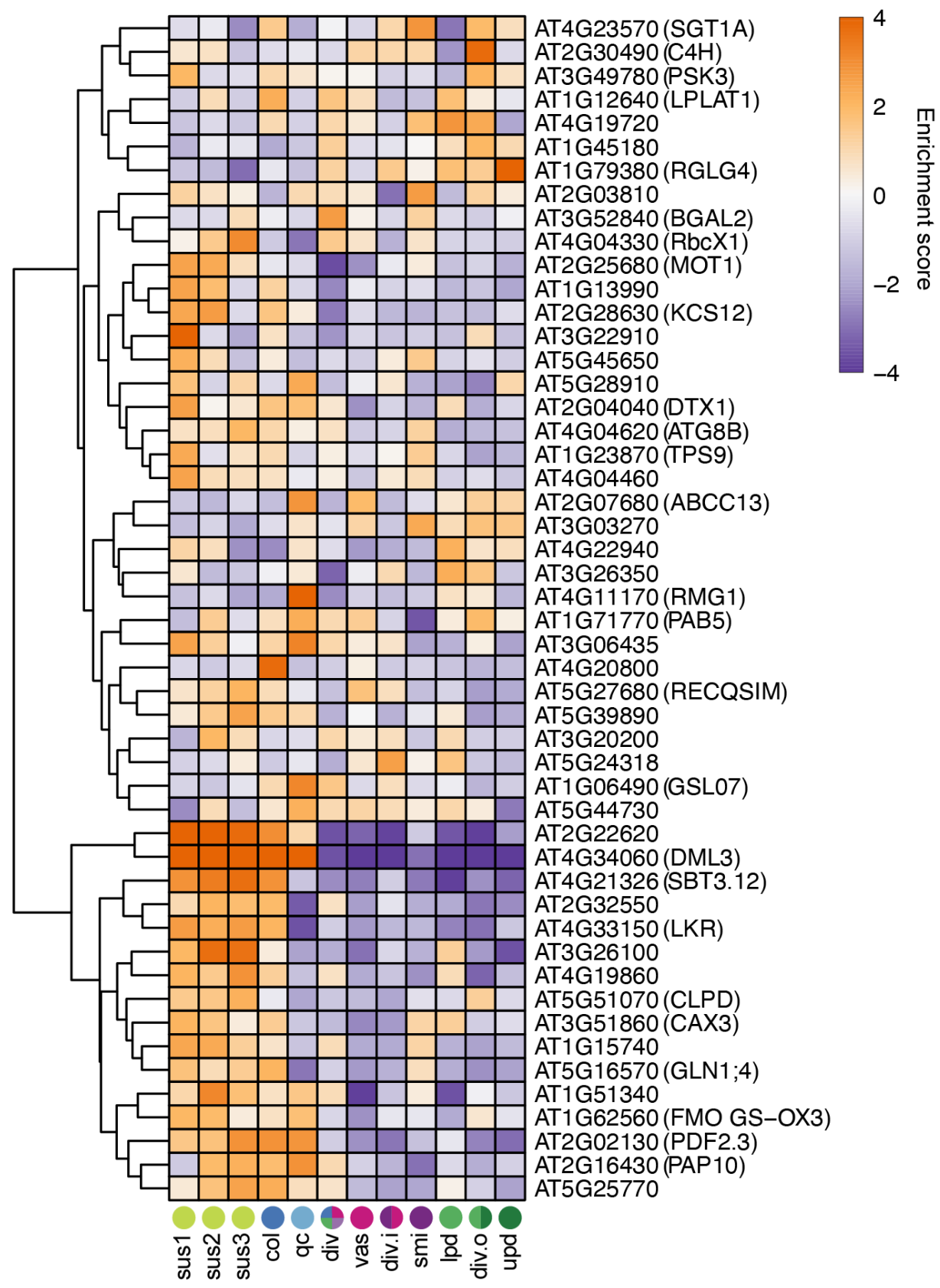

Figure S6. RDD target candidate expression across embryonic cell types.

Heatmap of enrichment scores for 50 ROS1/DML2/DML3 (RDD) targets detected in $\geq 10 \%$ nuclei in $\geq 1$ cluster and with enrichment scores $\geq 2$ in $\geq 1$ cluster. Enrichment scores are colored according to the key. Gene names are indicated and cluster identities are marked and color-coded at the bottom. Cluster identities are as in Fig. 2G. 


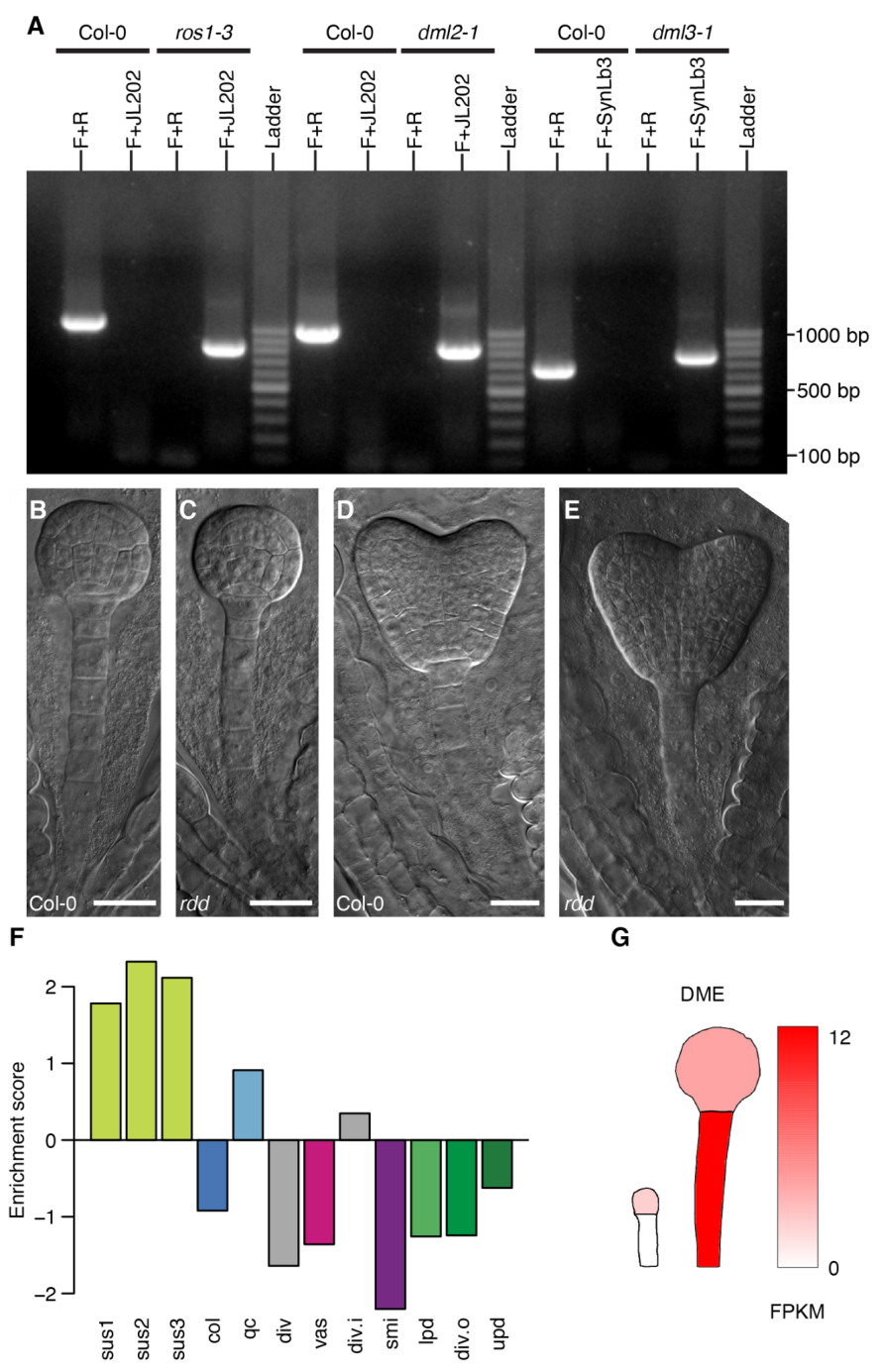

Figure S7. Phenotypic characterization of rdd mutant embryos and DME expression across embryonic cell types. (A) Genotyping PCR of characterized T-DNA insertions knock-out lines of ROS1, DML2 and DML3 in the ros1-3 dm/2-1 dm/3-1 (rdd) triple mutant (Penterman et al., 2007). Gene-specific forward "F" and reverse "R" primers are indicated, as well JL202 and SynLB3 primers used to detect T-DNA insertions. (B-E) Representative Nomarski images of Col-0 and rdd mutant globular $(B, C)$ and heart stage $(D, E)$ embryos. In total, 84 and 76 embryos were examined for Col-0 and rdd genotypes, respectively. Scale bars $=20 \mu \mathrm{m}$. (F) Bar plots illustrating enrichment scores of DME transcripts in 12 clusters corresponding to different embryonic cell types. Cluster identities are marked and color-coded at the bottom according to Fig. 2G. (G) Schematic representation of DME transcripts based on published mRNA-seq from apical and basal lineages in 1-cell and 32-cell stage embryos (Zhou et al, 2020). Transcript levels (FPKM; fragments per kilobase of transcript per million mapped reads) are colored according to the keys. 
Table S1. General information of snRNA-seq libraries and genes detected.

Click here to download Table S1

Table S2. Curated marker genes used for cell-type score calculation.

Click here to download Table S2

Table S3. Gene expression data and ranks.

Click here to download Table S3

Table S4. Gene ontology analyses results.

Click here to download Table S4

Table S5. Transcription factor motif correlations.

Click here to download Table S5

Table S6. Oligonucleotides used in this study.

Click here to download Table S6 